\title{
Lie algebra lattices and strings on $\mathrm{T}$-folds
}

\author{
Yuji Satoh $^{a}$ and Yuji Sugawara ${ }^{b}$ \\ ${ }^{a}$ Institute of Physics, University of Tsukuba, \\ Ibaraki 305-8571, Japan \\ ${ }^{b}$ Department of Physical Sciences, College of Science and Engineering, Ritsumeikan University, \\ Shiga 525-857\%, Japan \\ E-mail: ysatoh@het.ph.tsukuba.ac.jp, ysugawa@se.ritsumei.ac.jp
}

ABSTRACT: We study the world-sheet conformal field theories for T-folds systematically based on the Lie algebra lattices representing the momenta of strings. The fixed point condition required for the T-duality twist restricts the possible Lie algebras. When the T-duality acts as a simple chiral reflection, one is left with the four cases, $A_{1}, D_{2 r}, E_{7}, E_{8}$, among the simple simply-laced algebras. From the corresponding Englert-Neveu lattices, we construct the modular invariant partition functions for the T-fold CFTs in bosonic string theory. Similar construction is possible also by using Euclidean even self-dual lattices. We then apply our formulation to the T-folds in the $E_{8} \times E_{8}$ heterotic string theory. Incorporating non-trivial phases for the T-duality twist, we obtain, as simple examples, a class of modular invariant partition functions parametrized by three integers. Our construction includes the cases which are not reduced to the free fermion construction.

Keywords: Conformal Field Models in String Theory, String Duality, Superstring Vacua, Superstrings and Heterotic Strings

ARXIV EPRINT: 1611.08076 


\section{Contents}

1 Introduction $\quad 1$

2 Toroidal compactification and T-duality 3

3 Partition functions for T-folds 5

3.1 World-sheet partition functions 5

$\begin{array}{lll}3.2 & \text { Fixed points of T-duality transformations } & 6\end{array}$

$\begin{array}{lll}3.3 & \text { Fiber twist } & 7\end{array}$

4 T-folds from lattices $\quad 8$

4.1 Lie algebra lattices and Englert-Neveu lattices 8

$\begin{array}{lll}4.2 & \text { Euclidean even self-dual lattices } & 12\end{array}$

$\begin{array}{lll}4.3 & \text { Twists with phases } & 13\end{array}$

5 Application to heterotic string theory $\quad 13$

$\begin{array}{ll}5.1 \text { Orbifold action for heterotic T-folds } & 13\end{array}$

$\begin{array}{ll}5.2 \text { Construction of heterotic T-folds } & 16\end{array}$

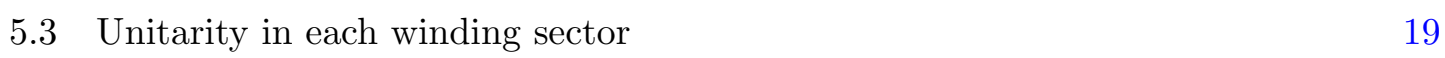

$\begin{array}{llr}6 & \text { Conclusions } & 20\end{array}$

A Summary of building blocks $\quad 21$

A.1 Building blocks associated with Lie algebra lattices 21

A.2 Free fermion chiral blocks 23

\section{Introduction}

A salient feature of string theory is that physics on different background geometries can be equivalent due to duality symmetries. This allows us to think of geometries whose coordinate patches are glued by duality transformations, as well as by ordinary general coordinate transformations [1-3]. They are relevant in understanding the vacua and the symmetries of string theory, and may give clues to formulations of string theories where the duality symmetries are manifest.

When such stringy geometries involve T-duality, they are called T-folds [4]. They have been studied mainly in the framework of supergravity and Double Field They [5]. In order to go beyond and analyze their quantum aspects, one may need the world-sheet approach based on conformal field theory (CFT). The transitions in the target space by T-duality are represented on the world-sheet as the twists by the T-duality transformations. Since 
they are generally left-right asymmetric, the world-sheet theories fall into a particular class of asymmetric orbifold CFTs. Such T-fold CFTs have been studied e.g. in [6-17].

As is generally the case for asymmetric orbifolds, the construction of the T-fold CFTs is not automatic. In addition to the modular invariance, there is an issue of the relative phases of the action of the T-duality twist on the left- and right-movers [7, 8], which may be regarded as an analog of the discrete torsions. As an interesting consequence of the explicit construction of a class of T-fold CFTs, it is found that T-folds provide a simple setting to realize non-supersymmetric string vacua with vanishing cosmological constant at least at one loop [16]. The mechanism there is extended to a more general class of asymmetric orbifolds [17]. Through T-folds and related more general non-geometric backgrounds (monodrofolds [3]), one can also explore the possibility that the world-sheet conformal interfaces [18-20], which may be regarded as fundamental from the world-sheet point of view, can be applied to string theory [14]. For the applications of the conformal interfaces to string theory, see e.g. [21-24].

In spite of the developments on T-fold CFTs so far, we are still lacking their general construction, which is in contrast to the quite general analysis from the target space point of view by supergravity. The purpose of this paper is to advance a step in this direction and provide a systematic construction of the modular invariant partition functions of $\mathrm{T}$ fold CFTs. A point of our construction is that we formulate the problem based on the momentum lattices in order to control the modular properties of the partition functions in the twisted sectors from the asymmetric T-duality twist. This allows us to consider the cases which are not reduced to the free fermion construction as well. ${ }^{1}$

The condition that the T-duality twist acts in a single Hilbert space requires the background moduli of the torus compactification to be invariant under the T-duality transformation, i.e. at the fixed points, as is found also in the supergravity analysis [1, 25]. Imposing this condition, we first give the modular invariant partition functions for T-folds in bosonic string theory, whose momentum lattices are associated with the Lie algebra lattices called Englert-Neveu lattices. The fixed-point condition restricts the possible Lie algebras. In the case where the T-duality acts as a simple chiral reflection in the right-mover, we are left with the four cases, $A_{1}, D_{2 r}, E_{7}, E_{8}$ among the simple simply-laced algebras. Similar construction is also possible by using Euclidean even self-dual lattices. We then apply our construction to the T-folds in the $E_{8} \times E_{8}$ heterotic string theory. Including non-trivial phases in the T-duality twists, the twisted partition functions in the originally intact leftmover are represented by the building blocks which appeared in the bosonic-string case. As simple examples, we explicitly construct a class of the modular invariant partition functions of the T-fold CFTs parametrized by three integers. The cases including the building blocks from $A_{1}$ and $E_{7}$ are not covered by the fermionization.

The rest of this paper is organized as follows: in section 2, we summarize the toroidal compactification and T-duality in bosonic string theory, which also serves as fixing our notation and conventions. In section 3, we set up our problem and analyze the fixed-point

\footnotetext{
${ }^{1}$ Based on the free fermion construction, systematic scans of of certain classes of T-fold CFTs have been performed for type II [10] and heterotic [11] superstrings.
} 
(self-duality) condition of the T-duality transformations. In section 4, we construct the modular invariant partition functions for T-folds in bosonic string theory, based on the Lie algebra lattices. In section 5 , we apply our construction to the T-folds in the heterotic string theory. In section 6 , we conclude with a summary and discussion. In appendix A, we summarize the characters of the affine Lie algebras and our building blocks for the modular invariant partition functions.

\section{Toroidal compactification and T-duality}

Let us consider the bosonic string theory where $d$ coordinates are compactified on a $d$ dimensional torus $T^{d}$. We basically follow the conventions in [26, 27]: the compactified coordinates $X^{i}(i=1, \ldots, d)$ have a periodicity $X^{i} \approx X^{i}+2 \pi$. The constant background fields, the metric $G_{i j}$ and the anti-symmetric tensor $B_{i j}$, are organized into a matrix,

$$
E_{i j}:=G_{i j}+B_{i j}
$$

which forms the background moduli of the compactification. The metric and anti-symmetric tensor are its symmetric and anti-symmetric part, respectively, i.e. $G=\left(E+E^{t}\right) / 2$, $B=\left(E-E^{t}\right) / 2$, where we have suppressed indices. The vielbeins are defined so that

$$
\sum_{a, b=1}^{d} \delta_{a b} e_{i}^{a} e_{j}^{b}=2 G_{i j}, \quad \sum_{a=1}^{d} e_{i}^{a} e_{a}^{* j}=\delta_{j}^{i},
$$

implying $\sum_{a, b=1}^{d} \delta^{a b} e_{a}^{* i} e_{b}^{* j}=\frac{1}{2} G^{i j}$. The space-time indices $i, j$ are converted to those of the tangent space $a, b$ by $e_{i}^{a} / \sqrt{2}, \sqrt{2} e_{a}^{* i}$, and they are lowered and raised by $G_{i j}, G^{i j}$ and $\delta_{a b}, \delta^{a b}$.

The world-sheet Hamiltonian takes the form,

$$
H=L_{0 L}+L_{0 R},
$$

where

$$
\begin{aligned}
L_{0 L} & :=\frac{1}{2} p_{L}^{2}+N, & L_{0 R} & :=\frac{1}{2} p_{R}^{2}+\tilde{N}, \\
p_{L a}(E) & :=e_{a}^{* i}\left[n_{i}-E_{i j} w^{j}\right], & p_{R a}(E) & :=e_{a}^{* i}\left[n_{i}+E_{i j}^{t} w^{j}\right],
\end{aligned}
$$

with $p_{L / R}^{2}=p_{L / R a} \delta^{a b} p_{L / R b}$ and $n_{i}, w^{j} \in \mathbb{Z}$ being the momentum and winding numbers. The dependence on the background $E_{i j}$ has been indicated explicitly in the momenta $p_{L / R}$. The transpose of $E_{i j}$ reads $E_{i j}^{t}=G_{i j}-B_{i j}$. The remaining terms $N, \tilde{N}$ are the number operators for the oscillator modes,

$$
N(E)=\sum_{m>0} \alpha_{-m}^{i}(E) G_{i j} \alpha_{m}^{j}(E), \quad \tilde{N}(E)=\sum_{m>0} \tilde{\alpha}_{-m}^{i}(E) G_{i j} \tilde{\alpha}_{m}^{j}(E),
$$

which take the values of non-negative integers. The partition function then takes the form

$$
\operatorname{Tr}\left[q^{L_{0 L}-\frac{d}{24}} \bar{q}^{L_{0 R}-\frac{d}{24}}\right]=\frac{1}{|\eta(\tau)|^{2 d}} \sum_{p_{L}, p_{R}} q^{\frac{1}{2} p_{L}^{2}} \bar{q}^{\frac{1}{2} p_{R}^{2}},
$$


where $q=e^{2 \pi i \tau}$ and $\tau=\tau_{1}+i \tau_{2}\left(\tau_{1} \in \mathbb{R}, \tau_{2}>0\right)$ is the modulus of the torus. The sum over the zero-modes are regarded as that over the Lorentzian lattice $\Lambda$ which is formed by the pair of the momenta $\left(p_{L}, p_{R}\right)$, and equipped with the Lorentzian inner product, $\left(p_{L}, p_{R}\right) \circ\left(p_{L}^{\prime}, p_{R}^{\prime}\right)=p_{L} p_{L}^{\prime}-p_{R} p_{R}^{\prime}$. Since this lattice is even self-dual, i.e. $\left(p_{L}, p_{R}\right)^{2} \in 2 \mathbb{Z}$ and $\Lambda=\Lambda^{*}$ (dual lattice), the above partition function is modular invariant. The zeromode part of $H$ is concisely expressed as

$$
H_{0}=\frac{1}{2}\left(p_{L}^{2}+p_{R}^{2}\right)=\frac{1}{2} v^{t} M(E) v
$$

where $v:=\left(w^{i}, n_{j}\right)^{t}$ and

$$
M(E):=\left(\begin{array}{cc}
G-B G^{-1} B & B G^{-1} \\
-G^{-1} B & G^{-1}
\end{array}\right) .
$$

This Hamiltonian keeps its form under the canonical map [27] associated with an $O(d, d, \mathbb{Z})$ matrix. To see this, we first note that an $O(d, d)$ matrix $g$ is defined as a $2 d \times 2 d$ matrix satisfying

$$
g^{t} J g=J, \quad J:=\left(\begin{array}{cc}
0 & I_{d} \\
I_{d} & 0
\end{array}\right),
$$

with $I_{k}$ being the $k$-dimensional identity matrix. One finds that $M(E) \in O(d, d, \mathbb{R})$. We then consider two backgrounds $E$ and $E^{\prime}$ which are related to each other by the $O(d, d, \mathbb{Z})$ transformation,

$$
E \rightarrow E^{\prime}=g(E):=(a E+b)(c E+d)^{-1},
$$

for $g \in O(d, d, \mathbb{Z})$ of the form,

$$
g=\left(\begin{array}{ll}
a & b \\
c & d
\end{array}\right) .
$$

This in turn implies the map of the metric,

$$
G \rightarrow G^{\prime}=\gamma_{L}^{\star} G \gamma_{L}^{-1}=\gamma_{R}^{\star} G \gamma_{R}^{-1}
$$

and that for the vielbein,

$$
e_{i}^{a} \rightarrow e_{i}^{\prime a}=e_{j}^{a}\left(\gamma_{L}^{-1}\right)_{j i}=e_{j}^{a}\left(\gamma_{R}^{-1}\right)_{j i}
$$

up to orthogonal transformations in the tangent space. Here, we have defined

$$
\gamma_{L}(E):=\left(d-c E^{t}\right), \quad \gamma_{R}(E):=(d+c E),
$$

and $O^{\star}:=\left(O^{t}\right)^{-1}$ for any invertible matrices, and used $\left(E^{\prime}\right)^{t}=\left(a E^{t}-b\right)\left(-c E^{t}+d\right)$. The canonical map acts on the oscillators as

$$
\alpha_{m}(E) \rightarrow \gamma_{L}^{-1}(E) \alpha_{m}\left(E^{\prime}\right), \quad \tilde{\alpha}_{m}(E) \rightarrow \gamma_{R}^{-1}(E) \tilde{\alpha}_{m}\left(E^{\prime}\right) .
$$

These are valid also for the zero-modes with the level $m=0$,

$$
\alpha_{0}(E)=\frac{1}{\sqrt{2}} G^{-1}(n-E w), \quad \tilde{\alpha}_{0}(E)=\frac{1}{\sqrt{2}} G^{-1}\left(n+E^{t} w\right),
$$


and thus (2.15) is translated into

$$
\hat{p}_{L}(E) \rightarrow \gamma_{L}^{t}(E) \hat{p}_{L}\left(E^{\prime}\right), \quad \hat{p}_{R}(E) \rightarrow \gamma_{R}^{t}(E) \hat{p}_{R}\left(E^{\prime}\right),
$$

in terms of

$$
\hat{p}_{L j}(E):=e_{i}^{a} p_{L a}(E)=\left(n-w E^{t}\right)_{j}, \quad \hat{p}_{R j}(E):=e_{i}^{a} p_{R a}(E)=(n+w E)_{j} .
$$

By these transformation rules, the number operators are mapped as

$$
N(E) \rightarrow N\left(E^{\prime}\right), \quad \tilde{N}(E) \rightarrow \tilde{N}\left(E^{\prime}\right),
$$

whereas

$$
H_{0}(E) \rightarrow \frac{1}{2} v^{t} g M(E) g^{t} v=H_{0}\left(E^{\prime}\right),
$$

where we have used $M\left(E^{\prime}\right)=g M(E) g^{t}$. These indeed show that the form of $H(E)$ is kept intact. The transformation (2.20) is regarded as either that of $M(E)$ with $v$ kept fixed or that of $v$ with $E$ kept fixed. Since $g \in O(d, d, \mathbb{Z})$ and hence the integer-valued vector $g^{t} v$ can be renamed as $v$, one confirms that the spectrum is invariant under the map.

\section{Partition functions for T-folds}

We now consider the asymmetric orbifolds by the $O(d, d, \mathbb{Z})$ T-duality transformations discussed in the previous section. In particular, we start from the target space,

$$
M \times \mathbb{R} \times T^{d},
$$

and twist the strings on it by the operator

$$
\sigma=\mathcal{T}_{2 \pi R} \otimes g
$$

Here $\mathcal{T}_{2 \pi R}$ stands for the shift by $2 \pi R$ in $\mathbb{R}$, and $g \in O(d, d, \mathbb{Z})$ for the T-duality twist acting on $T^{d}$. $M$ is other non-compact part. Consequently, we are considering a class of non-geometric backgrounds, i.e. T-folds, where $\mathbb{R}$ twisted by $\mathcal{T}_{2 \pi R}$ provides the 'base' circle $S_{R}^{1}$ with radius $R$, while $T^{d}$ is its 'fiber'.

\subsection{World-sheet partition functions}

In order to construct the world-sheet torus partition functions describing the strings on the above T-folds, we start with the partition function for the $\mathbb{R} \times T^{d}$ part with the $m$-fold temporal twist,

$$
Z_{(0, m)}(\tau):=\operatorname{Tr}\left[\sigma^{m} q^{L_{0}-\frac{c}{24}} \bar{q}^{\tilde{L}_{0}-\frac{c}{24}}\right] .
$$

Here, $L_{0}, \tilde{L}_{0}$ and $c$ are the Virasoro generators and the central charge, respectively, and the trace is taken over the untwisted Hilbert space. The trace in the base part is evaluated as

$$
\operatorname{Tr}_{\text {base }}\left[\left(\mathcal{T}_{2 \pi R}\right)^{m} q^{L_{0}^{\text {base }}-\frac{1}{24}} \tilde{q}^{\text {base }}-\frac{1}{24}\right]=Z_{R,(0, m)}(\tau)
$$


where

$$
Z_{R,(w, m)}(\tau)=\frac{R}{\sqrt{\tau_{2}}|\eta(\tau)|^{2}} e^{-\frac{\pi R^{2}}{\tau_{2}}|w \tau+m|^{2}}
$$

is the partition function for a free boson on $S_{R}^{1}$ in the winding sector with the spatial and temporal winding number $w, m \in \mathbb{Z}$, respectively. $\eta(\tau)$ is the Dedekind $\eta$ function. If the twist acted on the $\mathbb{R}$ and the $T^{d}$ part independently, the partition function in the base part would be $\sum_{w, m \in \mathbb{R}} Z_{R,(w, m)}$, giving the ordinary partition function for a compactified free boson. Denoting the fiber part as $Z_{(0, m)}^{T^{d}}$, the trace in $(3.3)$ is written as $Z_{(0, m)}=$ $Z_{R,(0, m)} Z_{(0, m)}^{T^{d}}$.

The partition functions in the base part transform covariantly under the modular transformations,

$$
\begin{aligned}
& \left.Z_{R,(w, m)}(\tau)\right|_{T}\left(\equiv Z_{R,(w, m)}(\tau+1)\right)=Z_{R,(w, w+m)}(\tau), \\
& \left.Z_{R,(w, m)}(\tau)\right|_{S}\left(\equiv Z_{R,(w, m)}(-1 / \tau)\right)=Z_{R,(m,-w)}(\tau) .
\end{aligned}
$$

These form a particular representation of the modular group. If the fiber part $Z_{(0, m)}^{T^{d}}$ satisfies the same form of the modular covariance,

$$
\left.Z_{(w, m)}^{T^{d}}(\tau)\right|_{T}=Z_{(w, w+m)}^{T^{d}}(\tau),\left.\quad Z_{(w, m)}^{T^{d}}(\tau)\right|_{S}=Z_{(m,-w)}^{T^{d}}(\tau),
$$

they give $Z_{(w, m)}^{T^{d}}$ with general winding numbers. Summing up all, the total partition function in such a case,

$$
Z(\tau)=Z_{M}(\tau) \sum_{w, m \in \mathbb{Z}} Z_{R,(w, m)}(\tau) Z_{(w, m)}^{T^{d}}(\tau)
$$

becomes modular invariant. Here, the first factor $Z_{M}$ is the contribution from $M$ in the background, which is assumed to be modular invariant itself.

In this argument, a non-trivial step for constructing the modular invariant $Z(\tau)$ is to find the fiber partition functions with the desired covariance (3.7). We see that a formulation based on the momentum lattices is useful to control the modular properties of the fiber part for that purpose.

\subsection{Fixed points of T-duality transformations}

In the fiber part, the twist operator $\sigma$ acts as a T-duality transformation. In general, T-duality connects different (but equivalent) world-sheet theories, and thus in order for the twist to be well-defined in a single Hilbert space, it has to be self-dual. ${ }^{2}$ In other words, the CFTs for T-folds are defined at the fixed points of the moduli space under the T-duality transformations. This also conforms to the supergravity analysis [1, 25]. Given the transformation rule (2.10), this condition is represented as

$$
E=(a E+b)(c E+d)^{-1},
$$

\footnotetext{
${ }^{2}$ In this paper, we use 'self-dual' to express both the 'self-dual' T-duality transformation satisfying (3.9) and the 'self-dual' lattice satisfying $\Lambda=\Lambda^{*}$ as below (2.6).
} 
for $g$ of the form (2.11). This also implies the invariance of the metric,

$$
G=\gamma_{L / R}^{t} G \gamma_{L / R}
$$

Denoting the momentum squared as

$$
p_{L}^{2}(E)=\hat{p}_{L}^{t}(E) \frac{1}{2} G^{-1} \hat{p}_{L}(E), \quad p_{R}^{2}(E)=\hat{p}_{R}^{t}(E) \frac{1}{2} G^{-1} \hat{p}_{R}(E),
$$

one finds that $p_{L / R}^{2}$ is separately invariant in the self-dual case.

To read off the form of the $O(d, d, \mathbb{Z})$ element implementing the self-dual transformation, we rewrite the transformation (2.17) in the form,

$$
\left(\begin{array}{l}
\hat{p}_{L j}(E) \\
\hat{p}_{R j}(E)
\end{array}\right)=P(E) v \rightarrow \Gamma(E)^{t} P(E) v=: P(E) g_{S D}^{t} v,
$$

where $v=\left(w^{i}, n_{j}\right)^{t}$ as before, $P(E):=\left(\begin{array}{cc}-E & I_{d} \\ E^{t} & I_{d}\end{array}\right)$, and $\Gamma(E):=\left(\begin{array}{cc}\gamma_{L} & 0 \\ 0 & \gamma_{R}\end{array}\right)$. After the map in the above, one has $\hat{p}_{L / R}(E)$ instead of $\hat{p}_{L / R}\left(E^{\prime}\right)$ due to the self-duality. Comparing this to the map of the Hamiltonian (2.20), one finds that $g_{S D}$ in the above represents the corresponding $O(d, d)$ element. Its explicit form is $[15,28]$

$$
\begin{aligned}
g_{S D} & =P^{t} \Gamma P^{\star} \\
& =\frac{1}{2}\left(\begin{array}{cc}
\gamma_{L}^{\star}+\gamma_{R}^{\star}-B \gamma_{-} G^{-1} & -\left(\gamma_{L}^{\star}+\gamma_{R}^{\star}-B \gamma_{-} G^{-1}\right) B-G \gamma_{-}+B \gamma_{+} \\
-\gamma_{-} G^{-1} & \gamma_{-} G^{-1} B+\gamma_{+}
\end{array}\right),
\end{aligned}
$$

with $\gamma_{ \pm}:=\gamma_{L} \pm \gamma_{R}$. Here, we have used $P(E)^{-1}=\frac{1}{2}\left(\begin{array}{cc}-I_{d} & I_{d} \\ E^{t} & E\end{array}\right)\left(\begin{array}{cc}G^{-1} & 0 \\ 0 & G^{-1}\end{array}\right)$, and the invariance of $G$ (3.10). This gives a necessary condition on the form of the self-dual transformation. Using the invariance of $G$, one can check that $g_{S D} \in O(d, d, \mathbb{R})$. Thus, if its components are integer-valued, $g_{S D}$ provides a proper $O(d, d, \mathbb{Z})$ self-dual transformation.

\subsection{Fiber twist}

As a simple example of (3.13), we consider in this paper the case where

$$
\gamma_{L}=I_{d}, \quad \gamma_{R}=-I_{d}
$$

and hence

$$
g_{S D}=-\left(\begin{array}{cc}
B G^{-1} & -B G^{-1} B+G \\
G^{-1} & -G^{-1} B
\end{array}\right)=-M(E) J .
$$

This is a $\mathbb{Z}_{2}$ element, $g_{S D}^{2}=I_{2 d}$, since $g^{-1}=J g^{t} J$ for $g \in O(d, d)$ and $M(E)^{t}=M(E)$. One can explicitly check that it induces a self-dual transformation $E \rightarrow E^{\prime}=E$. A sufficient condition for the integer-valuedness of $g_{S D}$ is $E_{i j}, G^{i j} / 2 \in \mathbb{Z}$, which follows from the product form in (3.13). When $E_{i j}$ is triangular e.g. $B_{i j}=G_{i j}(i>j)$, it is also sufficient 
that $E_{i j}, G^{i j} \in \mathbb{Z}$. This is confirmed by noting that $E_{i j} \in \mathbb{Z}$ implies $2 G_{i j}, G_{i i}$ (no sum) $\in \mathbb{Z}$ and that the products of $B_{i j}$ and matrices are rewritten as

$$
\sum_{j} B_{i j} M_{j k}=\sum_{j} G_{i j} M_{j k}-2 \sum_{i<j} G_{i j} M_{j k}-G_{i i} M_{i k}
$$

which is used for $M=G^{-1}$ or $G^{-1} B$. We note that in general the above $g_{S D}$ does not correspond to the $G \leftrightarrow G^{-1}(R \leftrightarrow 1 / R)$ duality, in spite of the forms of $\gamma_{L}, \gamma_{R}$.

In this case, from the transformation (2.15), i.e. $\left(\alpha_{m}, \tilde{\alpha}_{m}\right) \rightarrow\left(\alpha_{m},-\tilde{\alpha}_{m}\right)$, it follows that the oscillator contribution to the twisted partition function in the fiber part becomes $\left[\overline{2 \eta(\tau) / \theta_{2}(\tau)}\right]^{d / 2} \times \eta(\tau)^{-d}$. In the zero-mode part, the right momenta are projected out, $\hat{p}_{R}=0$, which implies that $n=-E^{t} w$ and hence we are left with the Euclidean lattice sum in the left-moving sector with this constraint. Taking into account $g_{S D}^{m}=1(m \in 2 \mathbb{Z})$ and $g_{S D}^{m}=g(m \in 2 \mathbb{Z}+1)$ in the untwisted sector $(w=0)$, we have

$$
Z_{(0, m)}^{T^{d}}(\tau)=\operatorname{Tr}\left[g_{S D}^{m} q^{L_{L 0}-\frac{d}{24}} \bar{q}^{L_{R 0}-\frac{d}{24}}\right]=\overline{\vartheta_{34}} d / 2(\tau) \cdot \frac{1}{\eta(\tau)^{d}} \sum_{w E \in \mathbb{Z}^{d}} q^{w^{t} G w},
$$

for $(m \in 2 \mathbb{Z}+1)$, where we have used $\theta_{2} \theta_{3} \theta_{4}=2 \eta^{3}$ and defined

$$
\vartheta_{p q}(\tau):=\frac{\theta_{p}(\tau) \theta_{q}(\tau)}{\eta(\tau)^{2}} .
$$

Below, we show that further choosing appropriate backgrounds yields the partition functions with the desired modular covariance (3.7), and thus the modular invariant total partition functions.

\section{T-folds from lattices}

In this section, we show that one can systematically construct the fiber partition functions with the desired modular covariance by choosing the background moduli $E_{i j}$ associated with the Lie algebra lattices, namely, sublattices of the weight lattice of a semi-simple Lie algebra. We first discuss the case of Englert-Neveu lattices [29] for simply-laced Lie algebras and then the case of Euclidean even self-dual lattices, both of which are straightforwardly realized by the momentum lattices of bosonic strings. For a review on the lattices in relation to string theory, see for example [30].

\subsection{Lie algebra lattices and Englert-Neveu lattices}

We consider the background with an affine symmetry of level one $\widehat{X}_{1}$ for a semi-simple simply-laced Lie algebra $X$ which is realized by [26, 31]

$$
E_{i j}=C_{i j}(i>j), \quad E_{i i}=\frac{1}{2} C_{i i}, \quad E_{i j}=0(i<j) .
$$

Here, $C_{i j}$ is the Cartan matrix of $X$, and the indices are not summed in the middle equation. The simple roots are normalized so that their norms are equal to two. In this background, $e_{i} \cdot e_{j}=2 G_{i j}=C_{i j}$ (for any $i, j$ ), and thus $e_{i}$ are the simple roots, whereas the duals $e^{* i}$ are 
the fundamental weights. Since $E_{i j} \in \mathbb{Z}$, the sum over the momenta in (2.4) becomes that over the weight lattice. Furthermore, since $p_{R a}-p_{L a}=e_{j}^{a} w^{j}$, the weights in the left- and the right-movers belong to the same conjugacy class. Up to this constraint, one can confirm by using the inverse of $P(E)$ in (3.12) that the summation reduces to the independent ones in each of the left- and right-movers. This gives an explicit realization of the Lorentzian even self-dual (Narain) lattice $\left(p_{L}, p_{R}\right)$ of the type called the Englert-Neveu lattice [29].

Thus, without twists, the relevant partition function is given by the sum of the diagonal combinations of of the level-one affine Lie algebra characters for $X$,

$$
\sum_{\alpha}\left|\chi_{\alpha}^{X}(\tau)\right|^{2}
$$

where

$$
\chi_{\alpha}^{X}(\tau):=\frac{1}{\eta(\tau)^{r}} \sum_{\boldsymbol{\lambda}_{\alpha} \in \Lambda_{(\alpha)}^{X}} q^{\frac{1}{2} \boldsymbol{\lambda}_{\alpha}^{2}},
$$

and $r$ is the rank of $X$. The summation is taken over the weights $\boldsymbol{\lambda}_{\alpha}$ belonging to a conjugacy class $\Lambda_{(\alpha)}^{X}$, i.e. an element of the coset $\Lambda_{X}^{*} / \Lambda_{X}$ labeled by $\alpha$, where $\Lambda_{X}^{*}$ and $\Lambda_{X}$ are the weight and the root lattice of $X$, respectively. A conjugacy class $\Lambda_{(\alpha)}^{X}$ also corresponds to an integrable representation of the affine Lie algebras at level one $\widehat{X}_{1}$.

For our purpose, a useful fact on the Lie algebra lattices is that these characters form a finite dimensional representation of the modular group, which is summarized as

$$
\left.\chi_{\alpha}^{X}(\tau)\right|_{T}=T_{\alpha \beta}^{X} \chi_{\beta}^{X}(\tau),\left.\quad \chi_{\alpha}^{X}(\tau)\right|_{S}=S_{\alpha \beta}^{X} \chi_{\beta}^{X}(\tau) .
$$

The modular matrices here are given by

$$
T_{\alpha \beta}^{X}=e^{-\pi i\left(\frac{r}{12}-\boldsymbol{\lambda}_{\beta}^{2}\right)} \delta_{\alpha \beta}, \quad S_{\alpha \beta}^{X}=\frac{1}{\sqrt{N_{c}}} e^{2 \pi i \boldsymbol{\lambda}_{\alpha} \cdot \boldsymbol{\lambda}_{\beta}},
$$

in terms of the weight vectors $\boldsymbol{\lambda}_{\alpha} \in \Lambda_{(\alpha)}^{X}$ and the number of the conjugacy classes $N_{c}$.

Now let us return to the construction of the partition functions for T-folds. First, we note that, since $E_{i j} \in \mathbb{Z}$, the constraint $w E \in \mathbb{Z}^{d}$ in (3.17) is automatically satisfied, and hence the summation becomes that over the root lattice. This enables us to utilize the above modular covariance to derive the partition functions in the twisted sectors.

Next, we note that the condition discussed in the previous section that $g_{S D}$ in (3.13) with $\gamma_{L / R}$ in (3.14) be integer-valued constrains the possible Englert-Neveu lattices. In particular, due to the condition that $G^{-1}=2 C^{-1}$ is integer-valued, we are left with

$$
A_{1}, \quad D_{r}(r: \text { even }), \quad E_{7}, \quad E_{8},
$$

among the simple simply-laced Lie algebras. Since $E_{i j}$ is triangular and its elements are integral, $E_{i j} \in \mathbb{Z}$, one finds that $g_{S D}$ is indeed integer-valued for the algebras in (4.6) and for their products, as discussed in section 3.3. Since any background realized by (4.1) is a fixed point under some non-trivial $O(d, d, \mathbb{Z})$ transformation $[26,32]$, one may consider other simply-laced Lie algebras. It may also be possible to consider the $\mathbb{Z}_{N}$ elements of $O(d, d, \mathbb{Z})$ as in $[15]$. However, the corresponding twists are more involved than (3.14). 
By starting from the twisted partition functions $Z_{(0, m)}^{T^{d}}$ given in (3.17) and using the modular properties (4.4), we can now uniquely determine the whole building blocks $Z_{(w, m)}^{T^{d}}$ for the cases (4.6) including the suitable phase factors to achieve the modular covariance (3.7). We concisely call this prescription and the resultant blocks as the 'modular completions $^{\prime 3}$ in the arguments below. Combining these $Z_{(w, m)}^{T^{d}}$ with other parts, we obtain the modular invariant partition functions of the form (3.8) for the T-fold CFTs. One can also utilize products $Z_{(w, m)}^{T^{d+d^{\prime}}}=Z_{(w, m)}^{T^{d}} \times Z_{(w, m)}^{T^{d^{\prime}}}$ where each factor corresponds to any of the algebras in (4.6).

We list the result of $Z_{(w, m)}^{T^{d}}$ in each case below. The corresponding Lie algebras are explicitly denoted there. Among the list, the cases for $D_{2}$ and $D_{4}$ appeared e.g. in $[16,17]$. The appearance of $A_{1}$ and $E_{7}$ may also be of interest, since such a case is not covered by the ordinary fermionization. We note that the action of $g_{S D}$ in the partition functions below is $\mathbb{Z}_{2}$ in the untwisted Hilbert space with $a=0$, which is in accord with the supergravity picture. In the twisted Hilbert spaces with $a \neq 0$, this is however not the case, except for $D_{r}(r \in 8 \mathbb{Z})$ and $E_{8}$. A related discussion on the modular covariance in the $A_{1}$ case is found in [34]. In the following, we denote the fiber torus corresponding to $X$ by $T^{d}[X]$.

Partition functions for $\boldsymbol{A}_{\mathbf{1}}$. There are two conjugacy classes $\Lambda_{(\alpha)}^{A_{1}}$ with $\alpha=0$, , which include the spin $\alpha / 2$-representation. The norms of the weight $\boldsymbol{\lambda}_{\alpha}$ for these conjugacy classes are $\boldsymbol{\lambda}_{0}^{2}=0, \boldsymbol{\lambda}_{1}^{2}=1 / 2(\bmod 2)$ and $\boldsymbol{\lambda}_{0} \cdot \boldsymbol{\lambda}_{1}=0(\bmod 1)$. Thus,

$$
T_{\alpha \beta}^{A_{1}}=e^{-\frac{\pi}{12} i} \operatorname{diag}(1, i), \quad S_{\alpha \beta}^{A_{1}}=\frac{1}{\sqrt{2}}\left(\begin{array}{cc}
1 & 1 \\
1 & -1
\end{array}\right) .
$$

Since $Z_{(0, m)}^{T^{1}\left[A_{1}\right]}(\tau)={\overline{\vartheta_{34}(\tau)}}^{1 / 2} \chi_{0}^{A_{1}}(\tau)(m \in 2 \mathbb{Z}+1)$, the modular completion yields

$$
Z_{(a, b)}^{T^{1}\left[A_{1}\right]}(\tau)= \begin{cases}\left|\chi_{0}^{A_{1}}(\tau)\right|^{2}+\left|\chi_{1}^{A_{1}}(\tau)\right|^{2} & (a \in 2 \mathbb{Z}, b \in 2 \mathbb{Z}), \\ e^{\frac{\pi i}{8} a b^{3}} \frac{1}{\vartheta_{34}(\tau)} \frac{1}{2} \cdot \frac{1}{2}\left[\chi_{+}^{A_{1}}(\tau)+i^{a} \chi_{-}^{A_{1}}(\tau)\right] & (a \in 2 \mathbb{Z}, b \in 2 \mathbb{Z}+1), \\ e^{-\frac{\pi i}{8} a^{3} b \overline{\vartheta_{23}(\tau)} \frac{1}{2}} \cdot \frac{1}{\sqrt{2}}\left[\chi_{0}^{A_{1}}(\tau)+i^{b} \chi_{1}^{A_{1}}(\tau)\right] & (a \in 2 \mathbb{Z}+1, b \in 2 \mathbb{Z}), \\ e^{-\frac{\pi i}{8} a^{3} b \overline{\vartheta_{24}(\tau)} \frac{1}{2}} \cdot \frac{1}{\sqrt{2}}\left[\chi_{0}^{A_{1}}(\tau)+i^{a+b-1} \chi_{1}^{A_{1}}(\tau)\right] & (a \in 2 \mathbb{Z}+1, b \in 2 \mathbb{Z}+1),\end{cases}
$$

which indeed satisfies the desired modular covariance (3.7). Here, $\chi_{ \pm}^{A_{1}}:=\chi_{0}^{A_{1}} \pm \chi_{1}^{A_{1}}$, and the explicit forms of the characters $\chi_{0,1}^{A_{1}}$ are found in (A.6) in the appendix.

Partition functions for $\boldsymbol{D}_{\boldsymbol{r}}\left(\boldsymbol{r}\right.$ : even). There are four conjugacy classes $\Lambda_{(\alpha)}^{D_{r}}$, which include the vacuum, vector, spinor or conjugate-spinor representation. We label these by $\alpha=0, v, s, c$, respectively. A representative weight in each conjugacy class is $(0, \ldots, 0)$, $(1,0, \ldots, 0),( \pm 1 / 2, \pm 1 / 2, \ldots)$ with even or odd number of minus signs. Thus,

$$
T_{\alpha \beta}^{D_{r}}=e^{-\frac{\pi i}{12} r} \operatorname{diag}\left(1,-1, e^{\frac{\pi i}{4} r}, e^{\frac{\pi i}{4} r}\right), \quad S_{\alpha \beta}^{D_{r}}=\frac{1}{2}\left(\begin{array}{cccc}
1 & 1 & 1 & 1 \\
1 & 1 & -1 & -1 \\
1 & -1 & i^{r} & -i^{r} \\
1 & -1 & -i^{r} & i^{r}
\end{array}\right) .
$$

\footnotetext{
${ }^{3}$ In this paper we use this terminology in the sense different from e.g. [33].
} 
Since $Z_{(0, m)}^{T^{r}\left[D_{r}\right]}(\tau)={\overline{\vartheta_{34}(\tau)}}^{r / 2} \chi_{0}^{D_{r}}(\tau)(m \in 2 \mathbb{Z}+1)$, the modular completion yields

$$
Z_{(a, b)}^{T^{r}\left[D_{r}\right]}(\tau)= \begin{cases}\frac{1}{2|\eta(\tau)|^{2 r}}\left[\left|\theta_{3}(\tau)\right|^{2 r}+\left|\theta_{4}(\tau)\right|^{2 r}+\left|\theta_{2}(\tau)\right|^{2 r}\right] & (a \in 2 \mathbb{Z}, b \in 2 \mathbb{Z}), \\ e^{\frac{\pi i r}{8} a b} \frac{\vartheta_{34}(\tau)^{\frac{r}{2}}}{2 \eta(\tau)^{r}}\left[\theta_{3}(\tau)^{r}+e^{\frac{\pi i r}{4} a} \theta_{4}(\tau)^{r}\right] & (a \in 2 \mathbb{Z}, b \in 2 \mathbb{Z}+1), \\ e^{-\frac{\pi i r}{8} a b} \overline{\vartheta_{23}(\tau)^{\frac{r}{2}}} \cdot \frac{1}{2 \eta(\tau)^{r}}\left[\theta_{3}(\tau)^{r}+e^{\frac{\pi i r}{4} b} \theta_{2}(\tau)^{r}\right] & (a \in 2 \mathbb{Z}+1, b \in 2 \mathbb{Z}), \\ e^{-\frac{\pi i r}{8} a b} \frac{r}{\vartheta_{24}(\tau)^{\frac{r}{2}}} \cdot \frac{1}{2 \eta(\tau)^{r}}\left[\theta_{4}(\tau)^{r}+e^{\frac{\pi i r}{4}(a+b-1)} \theta_{2}(\tau)^{r}\right] & (a \in 2 \mathbb{Z}+1, b \in 2 \mathbb{Z}+1),\end{cases}
$$

satisfying the modular covariance (3.7). The theta functions are related to the $D_{r}$ characters as in (A.8).

Partition functions for $\boldsymbol{E}_{\mathbf{7}}$. There are two conjugacy classes $\Lambda_{(\alpha)}^{E_{7}}$, which include the vacuum or the 56 representation. We label these by $\alpha=0$ and $\alpha=1$, respectively. The norms of the corresponding weights are $\boldsymbol{\lambda}_{0}^{2}=0, \boldsymbol{\lambda}_{1}^{2}=3 / 2(\bmod 2)$ and $\boldsymbol{\lambda}_{0} \cdot \boldsymbol{\lambda}_{1}=0$ $(\bmod 1)$. Thus,

$$
T_{\alpha \beta}^{E_{7}}=e^{-\frac{7 \pi}{12} i} \operatorname{diag}(1,-i), \quad S_{\alpha \beta}^{E_{7}}=\frac{1}{\sqrt{2}}\left(\begin{array}{cc}
1 & 1 \\
1 & -1
\end{array}\right) .
$$

Since $Z_{(0, m)}^{T^{7}\left[E_{7}\right]}(\tau)={\overline{\vartheta_{34}(\tau)}}^{7 / 2} \chi_{0}^{E_{7}}(\tau)(m \in 2 \mathbb{Z}+1)$, the modular completion yields

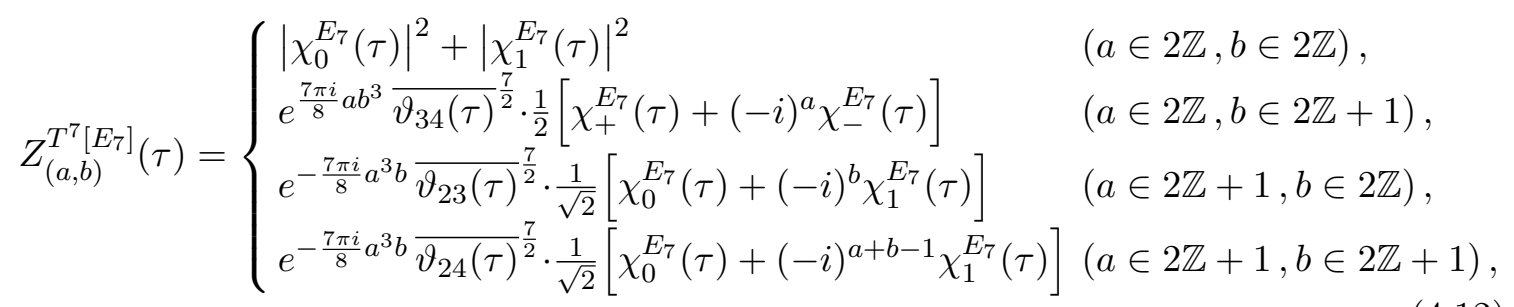

satisfying the modular covariance (3.7). Here , $\chi_{ \pm}^{E_{7}}:=\chi_{0}^{E_{7}} \pm \chi_{1}^{E_{7}}$, and the explicit forms of the characters are found in (A.9) and (A.10).

Partition functions for $\boldsymbol{E}_{\mathbf{8}}$. There is only one conjugacy class including the vacuum representation, which we label by $\alpha=0$. The norms of the weights is $\lambda_{0}^{2}=0(\bmod 2)$. This is an even self-dual lattice and hence the modular property is trivial up to the phases coming from the eta functions for the oscillator part,

$$
T_{\alpha \beta}^{E_{8}}=e^{-\frac{8 \pi}{12} i}, \quad S_{\alpha \beta}^{E_{8}}=1 .
$$

Since $Z_{(0, m)}^{T^{8}\left[E_{8}\right]}(\tau)={\overline{\vartheta_{34}(\tau)}}^{4} \chi_{0}^{E_{8}}(\tau)(m \in 2 \mathbb{Z}+1)$, the modular completion yields

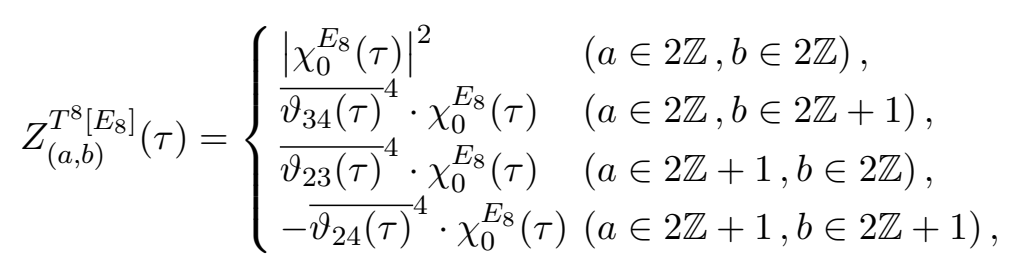

satisfying the modular covariance (3.7). The explicit form of the character $\chi_{0}^{E_{8}}(\tau)$ is given in (A.12). 


\subsection{Euclidean even self-dual lattices}

Another class of the lattices for which the modular properties of the Euclidean lattice sum in (3.17) are well controlled is those associated with Euclidean even self-dual lattices. Precisely, we start from a Lorentzian lattice $\left(p_{L}, p_{R}\right)$ which is specified by the basis $e^{* i}$ of a Euclidean even self-dual lattice. The vielbein $e_{i}$ and the metric $G_{i j}$ are determined by (2.2). The matrix corresponding to the Cartan matrix is defined in this case by $C_{i j}=2 G_{i j}$, which fixes the background moduli $E_{i j}$ by adopting the relations (4.1). With this setting, after the T-duality twist we are left with the sum over the Euclidean even self-dual lattice in (3.17). ${ }^{4}$

The Euclidean even self-dual lattices are allowed only for dimensions $d \in 8 \mathbb{Z}$. At $d=8$, the unique lattice is the $E_{8}$ lattice, which is already discussed in the previous subsection. At $d=16$, there are two. One is the $E_{8} \times E_{8}$ lattice and the other is the $\operatorname{Spin}(32) / \mathbb{Z}_{2}$ lattice. At $d=24$, there are twenty four. These are called Niemeier lattices.

For an even-self dual lattice, $e^{* i}$ and $e_{i}$ span the same lattice since it is self-dual. Moreover $e^{* i} \cdot e^{* j}, e_{i} \cdot e_{j} \in \mathbb{Z}(i \neq j)$ and they are even for $i=j$, since it is even. Thus, $G^{i j} / 2, E_{i j} \in \mathbb{Z}$ and the integer-valuedness of $g_{S D}$ for (3.14) is satisfied.

The even self-duality also means that the modular property of the lattice sum is trivial,

$$
T_{\alpha \beta}^{E S D}=e^{-\frac{\pi d}{12} i}, \quad S_{\alpha \beta}^{E S D}=1,
$$

as in the $E_{8}$ case. Thus, denoting the corresponding character by $\chi^{E S D}$, the fiber partition function for a $d$-dimensional even self-dual lattice reads

$$
Z_{(a, b)}^{T^{d}[E S D]}(\tau)= \begin{cases}\frac{\left|\chi^{E S D}(\tau)\right|^{2}}{\vartheta_{34}(\tau)} d / 2 & (a \in 2 \mathbb{Z}, b \in 2 \mathbb{Z}), \\ \frac{\vartheta_{23}(\tau)}{d / 2} \cdot \chi^{E S D}(\tau) & (a \in 2 \mathbb{Z}, b \in 2 \mathbb{Z}+1), \\ (-1)^{d / 8} \frac{\vartheta_{24}(\tau)}{d / 2} \cdot \chi^{E S D}(\tau) & (a \in 2 \mathbb{Z}+1, b \in 2 \mathbb{Z}+1),\end{cases}
$$

satisfying the modular covariance (3.7). The explicit forms of $\chi^{E S D}$ 's are found by using the relation of these even-self dual lattices and Lie algebra lattices [30]. For example for $d=16$, the $\operatorname{Spin}(32) / \mathbb{Z}_{2}$ lattice is realized as the $D_{16}$ sublattices with the vacuum and the spinor conjugacy class, and thus

$$
\chi^{E S D}(\tau)=\chi^{\operatorname{Spin}(32) / \mathbb{Z}_{2}}(\tau)=\chi_{0}^{D_{16}}(\tau)+\chi_{s}^{D_{16}}(\tau),
$$

in this case. Furthermore, by the identity of the Eisenstein series $E_{8}(\tau)=E_{4}(\tau)^{2}$, one has $\chi^{\operatorname{Spin}(32) / \mathbb{Z}_{2}}=\left[\chi_{0}^{E_{8}}(\tau)\right]^{2}=\chi^{E_{8} \times E_{8}}$.

As in the case of the Englert-Neveu lattices, combining these with other parts, we obtain the modular invariant partition functions of the form (3.8) for the T-fold CFTs. The action of $g_{S D}$ on $Z_{(a, b)}^{T^{d}[E S D]}$ in this case is $\mathbb{Z}_{2}$ both in the untwisted and twisted Hilbert spaces.

\footnotetext{
${ }^{4}$ A typical example of Euclidean odd self-dual lattices is $\mathbb{Z}^{n}$, which is also unique for dimensions $n \leq 8$ (see e.g. [35]). It is also realized as the $D_{n}$ lattice with the conjugacy classes (0) and (1). However, the partition function is not compatible with the modular covariance of the form (3.6): starting with $Z_{(0,1)}^{T^{n}}=\bar{\vartheta}_{34}^{n / 2} \theta_{3}^{n} / \eta^{n}$, and assuming the covariance (3.6), successive transformations $\operatorname{STSTS}(\neq 1)$ would give $Z_{(0,1)}^{T^{n}}=\bar{\vartheta}_{34}^{n / 2} \theta_{4}^{n} / \eta^{n}$, in contradiction. Choosing the basis so that $e_{i} \cdot e_{j}=C_{i j}=\delta_{i j}$, the integer-valuedness of $g_{S D}$ is not satisfied either if we adopt (4.1) and (3.14).
} 


\subsection{Twists with phases}

In acting with the T-duality transformation, the relative phase between the left and the right mover are not unique. Such a phase is strongly constrained when one requires that the full operator product expansion, not only the chiral one, of the vertex operators respects the invariance under the twist $[7,15]$. For the $A_{1}$ lattice, the phase in this case becomes $(-1)^{n w}$, with which the T-duality acts as an inner automorphism of $\mathfrak{s u}(2)_{L} \oplus \mathfrak{s u}(2)_{R}[7,8]$. In section 5 , the possibility of including such phases is explicitly discussed, when we apply our construction to the T-fold CFTs for the heterotic string. In the partition function, the above phase is implemented by the shift $\tau \rightarrow \tau+1 / 2$ since $p_{L}^{2}-p_{R}^{2} \in 2 \mathbb{Z}$. It would be an interesting problem if the phases in higher dimensional cases [15] are also interpreted from the current algebra or the lattice point of view.

\section{Application to heterotic string theory}

So far, we have discussed T-folds in bosonic string theory. Our construction can be applied straightforwardly to the case of superstrings. In particular, applying the results of the Englert-Neveu lattices for $D_{2}, D_{4}$ to type II superstrings reduces to the analysis in [16]. Its generalization has also been discussed [17]. A notable point in these analyses is that, combined with further twists, our T-fold CFTs simply realize the non-supersymmetric vacua with vanishing cosmological constant at least at one loop.

In this section, we apply our construction to the heterotic string theory. In our set up, the left-mover is the bosonic string with the $E_{8} \times E_{8}$-lattice, while the right-mover is the superstring including the fermionic one. We focus on the supersymmetric models preserving 8 space-time supercharges. Namely, we assume that the chiral reflection acts on the right-movers along a four dimensional fiber torus, which we choose to be $T^{4}\left[D_{4}\right]$ for simplicity. We briefly comment on the case $T^{4}\left[D_{2} \times D_{2}\right] \equiv T^{4}\left[\left(A_{1}\right)^{4}\right]$ later on. The T-fold CFTs for the heterotic string have been discussed e.g. in $[6,11]$.

However, since we are considering asymmetric orbifolds, we still have a large variety of possibilities for the heterotic vacua: the orbifold group may act non-trivially on $(i)$ the left-mover of $T^{4}\left[D_{4}\right]$ and (ii) the 16-dim. internal torus with the $E_{8} \times E_{8}$ lattice, while maintaining the modular invariance. We demonstrate how we can systematically construct the modular invariants describing a large number of such heterotic string vacua, by utilizing the modular covariant blocks (4.8), (4.10), (4.12) given in section 4. Above all, we uncover a fairly non-trivial phase factor that realizes the manifest modular covariance of the total building blocks.

\subsection{Orbifold action for heterotic T-folds}

Let us elaborate on a concrete construction of the models of heterotic T-folds. We start with the $E_{8} \times E_{8}$ heterotic string compactified on $T^{4}\left[D_{4}\right]\left(X^{6, \ldots, 9}\right.$-directions). As in the previous section, we consider the orbifolding by $\sigma \equiv \mathcal{T}_{2 \pi R} \otimes g$. Here, $g$ acts along the $T^{4}$-direction as the chiral reflection,

$$
g: X_{R}^{i} \longmapsto-X_{R}^{i}, \quad \psi_{R}^{i} \longmapsto-\psi_{R}^{i}, \quad(i=6,7,8,9),
$$




\begin{tabular}{|c|c|c|c|c|c|c|c|c|c|}
\hline$r$ & 0 & 1 & 2 & 3 & 4 & 5 & 6 & 7 & 8 \\
\hline$X_{r}$ & trivial & $A_{1}$ & $D_{2}$ & $A_{1} D_{2}$ & $D_{4}$ & $A_{1} D_{4}$ & $D_{6}$ & $E_{7}$ & $E_{8}$ \\
\hline
\end{tabular}

Table 1. list of $X_{r}$.

which preserves $1 / 2$-SUSY, while $\mathcal{T}_{2 \pi R}$ denotes the shift operator acting on the 'base' $X^{5}$ direction,

$$
\mathcal{T}_{2 \pi R}: X^{5} \equiv X_{L}^{5}+X_{R}^{5} \longmapsto X^{5}+2 \pi R .
$$

We use the notation $Z_{R,(w, m)}(\tau)$ defined in (3.5) to write down the partition function for the $X^{5}$-direction.

We further allow $g$ to act non-trivially on the left-mover as the 'chiral shifts' along various compact directions characterized by three integers $\left(r_{1}, r_{2}, r_{3}\right)$, where $r_{1}(\leq 4), r_{2}, r_{3}(\leq 8)$ are associated with $T^{4}\left[D_{4}\right]$ and the two $E_{8}$-directions. Requiring the modular covariance, it turns out that this orbifold action provides extra phases mentioned in section 4.3. We now separately specify the orbifold action $g$ on these sectors.

Action on left-mover of $T^{4}\left[D_{4}\right]$-direction. As mentioned above, $g$ acts as the chiral reflection $\left(-\mathbf{1}_{R}\right)^{\otimes 4}$ for the right-mover.

To specify the left-moving action, we consider the decomposition of the conjugacy classes of $D_{4}$ for a fixed integer $r_{1}\left(0 \leq r_{1} \leq 4\right)$,

$$
\Lambda_{(\alpha)}^{D_{4}}=\sum_{\left\{\alpha_{i}\right\}, \beta}\left[\Lambda_{\left(\alpha_{1}\right)}^{A_{1}} \oplus \cdots \oplus \Lambda_{\left(\alpha_{r_{1}}\right)}^{A_{1}}\right] \oplus \Lambda_{(\beta)}^{X_{4-r_{1}}}
$$

where $\alpha=0, v, s, c$ for $D_{4}$, and $\alpha=0,1$ for $A_{1}$ as in section 4.1. We also denote by $\alpha=0$ the conjugacy class for the basic representation (the root lattice itself) for any algebra $X$, i.e. $\Lambda_{(0)}^{X} \equiv \Lambda_{X}$. We can uniquely determine the (semi-simple) Lie algebra $X_{4-r_{1}}$ of rank $4-r_{1}$ on the R.H.S. by imposing the following conditions;

(i) $X_{4-r_{1}}$ is composed only of the irreducible components given in (4.6), that is, $A_{1}, D_{r}$ ( $r$ : even), $E_{7}, E_{8}$.

(ii) $X_{4-r_{1}}$ is 'maximal' in the following sense; when taking $\Lambda_{(0)}^{D_{4}} \equiv \Lambda_{D_{4}}$ on the L.H.S of (5.3), there is only one component with $\alpha_{1}=\cdots=\alpha_{r_{1}}=0$ on the R.H.S, which should inevitably couple with $\Lambda_{(0)}^{X_{4-r_{1}}}$. (In other words, the conjugacy class $\Lambda_{(\beta)}^{X_{4-r_{1}}}$ with $\beta \neq 0$ always couples with at least one spin 1/2-representation in the $r_{1}$ factors of $A_{1}$.)

We explicitly exhibit the solutions of $X_{r}$ in table 1 for $r \leq 4$. The entries for $r \geq 5$ in this table are used shortly in the discussion on the $E_{8}$-part.

Then, we define the left-moving action of $g$ as an involution $\left.g\right|_{\text {left-mover }}=\left[\rho_{A_{1}}\right]^{\otimes r_{1}}$ associated with the lattice component $\left[\Lambda_{(*)}^{A_{1}}\right]^{r_{1}}$, where $\rho_{A_{1}}$ is an involutive (outer-)automorphism 
acting on the $\widehat{A}_{1}$-currents $\left\{J^{a}\right\}(a=1,2,3)$ as $^{5}$

$$
\rho_{A_{1}} J^{a}(z) \rho_{A_{1}}^{-1}=-J^{a}(z), \quad(a=1,2), \quad \rho_{A_{1}} J^{3}(z) \rho_{A_{1}}^{-1}=J^{3}(z) .
$$

This operator is actually interpreted as the chiral half-shift along the direction of lattice $\Lambda_{(*)}^{A_{1}}$ (up to some phase factor), when the $\widehat{A}_{1}$-currents $J^{a}$ are bosonized in the standard fashion.

We next consider the relevant partition sum with the orbifold twist $g$ inserted. To this end, we recall that $g$ acts on the right-mover as the chiral reflection $\left(-\mathbf{1}_{R}\right)^{\otimes 4}$, which leaves the sum over the root lattice in the left-mover as in (3.17). Together with the condition (ii) given above as well as the definition of $\rho_{A_{1}}$, it is then obvious that only the basic representation of $\left[\widehat{A}_{1}\right]^{r_{1}} \oplus \widehat{X}_{4-r_{1}}$ can yield non-vanishing contributions. The right-mover just gives $\overline{\left[\vartheta_{34}(\tau)^{1 / 2}\right]^{4}}$, as already described in section 4 . On the other hand, the $\left[\rho_{A_{1}}\right]^{\otimes r_{1}}$ twist in the left-mover acts as sign factors on the relevant charge lattice, while leaving the oscillator parts unchanged, which again provides $\left[\vartheta_{34}(\tau)^{1 / 2}\right]^{r_{1}}$ eventually. (See e.g. [8] for detail.) In this way, we obtain

$$
\begin{aligned}
\operatorname{Tr}_{T^{4}\left[D_{4}\right]}\left[g q^{L_{0}-\frac{4}{24}} \overline{q^{\tilde{L}_{0}-\frac{4}{24}}}\right] & =\overline{\vartheta_{34}(\tau)^{2}} \cdot \vartheta_{34}(\tau)^{\frac{r_{1}}{2}} \chi_{0}^{X_{4-r_{1}}}(\tau) \\
& \equiv\left[\overline{\tilde{\chi}_{(0,1)}^{A_{1}}(\tau)}\right]^{4} \cdot\left[\tilde{\chi}_{(0,1)}^{A_{1}}(\tau)\right]^{r_{1}} \chi_{(0,1)}^{X_{4-r_{1}}(\tau) .}
\end{aligned}
$$

Here, the building blocks $\chi_{(0,1)}^{X_{r}}(\tau) \equiv \chi_{0}^{X_{r}}(\tau)$ from the lattice and $\tilde{\chi}_{(0,1)}^{A_{1}}(\tau) \equiv \vartheta_{34}(\tau)^{1 / 2}$ for the $\rho_{A_{1}}$-twist already appeared in (4.8), (4.10), (4.12), and are summarized in appendix A.1 explicitly. For later convenience, we have also rewritten $\bar{\vartheta}_{34}^{1 / 2}$ in the right-mover as ${\overline{\tilde{\chi}_{(0,1)}}}_{A_{1}}$, although it does not necessarily originate from the $\hat{A}_{1}$-symmetry.

For example, in the case of $r_{1}=1$, the relevant decomposition is

$$
\Lambda_{D_{4}}=\Lambda_{(0)}^{A_{1}} \oplus \Lambda_{(0)}^{X_{3}}+\cdots \equiv \Lambda_{A_{1}} \oplus \Lambda_{A_{1}} \oplus \Lambda_{D_{2}}+\cdots
$$

and the trace (5.5) becomes

$$
\left[\overline{\tilde{\chi}_{(0,1)}^{A_{1}}(\tau)}\right]^{4} \cdot \tilde{\chi}_{(0,1)}^{A_{1}}(\tau) \chi_{(0,1)}^{X_{3}}(\tau) \equiv \frac{\overline{\theta_{3}^{2} \theta_{4}^{2}}}{\eta^{4}} \cdot\left[\frac{\theta_{3} \theta_{4}}{\eta^{2}}\right]^{\frac{1}{2}} \chi_{0}^{A_{1}}(\tau) \chi_{0}^{D_{2}}(\tau),
$$

where $\chi_{0}^{A_{1}}(\tau), \chi_{0}^{D_{2}}(\tau)$ are the characters of basic representations of $\left(\widehat{A}_{1}\right)_{1},\left(\widehat{D}_{2}\right)_{1}$ respectively.

\footnotetext{
${ }^{5} \rho_{A_{1}}$ is explicitly written as

$$
\rho_{A_{1}}=e^{-i \pi \frac{\ell}{2}} e^{i \pi J_{0}^{3}}
$$

on the integrable representation of spin $\ell / 2(\ell=0,1)$. The phase factor $e^{-i \pi \frac{\ell}{2}}$ is necessary to make $\rho_{A_{1}}$ involutive. Note that the simpler inner-automorphism $\tilde{\rho}_{A_{1}} \equiv e^{i \pi J_{0}^{3}}$ is not involutive;

$$
\left[\tilde{\rho}_{A_{1}}\right]^{2}=(-1)^{\ell}
$$

which would play the role of the ' $\mathbb{Z}_{4}$-chiral reflection' appearing in $[16,17]$. It is presumably an interesting possibility to extend the heterotic vacua given in this section so as to include the $\mathbb{Z}_{4}$-action $\tilde{\rho}_{A_{1}}$, and we would like to discuss it elsewhere.
} 
Action on two $\boldsymbol{E}_{8}$-directions. Let us first focus on one of the $E_{8}$-factors. There, we have a unique conjugacy class, that is, the root lattice itself. We fix an integer $r_{2}$ $\left(0 \leq r_{2} \leq 8\right)$, and consider the decomposition of the root lattice $\Lambda_{E_{8}}$ as

$$
\Lambda_{E_{8}}=\sum_{\left\{\alpha_{i}\right\}, \beta}\left[\Lambda_{\left(\alpha_{1}\right)}^{A_{1}} \oplus \cdots \oplus \Lambda_{\left(\alpha_{r_{2}}\right)}^{A_{1}}\right] \oplus \Lambda_{(\beta)}^{X_{8-r_{2}}}
$$

The decomposition (5.8) is again uniquely determined by essentially the same conditions as for $T^{4}\left[D_{4}\right]$, i.e. (i) and (ii) with $D_{4}, X_{4-r_{1}}$ replaced by $E_{8}, X_{8-r_{1}}$, respectively. The result of $X_{8-r_{2}}$ is listed in table $1 .{ }^{6}$

We then define the $g$-action in this sector by $\left[\rho_{A_{1}}\right]^{\otimes r_{2}}$ associated with the lattice component $\left[\Lambda_{(*)}^{A_{1}}\right]^{r_{2}}$. Since the relevant trace has contribution only from the basic representation of $X_{8-r_{2}}$, we have

$$
\operatorname{Tr}_{E_{8}}\left[g q^{L_{0}-\frac{8}{24}}\right]=\left[\tilde{\chi}_{(0,1)}^{A_{1}}(\tau)\right]^{r_{2}} \chi_{(0,1)}^{X_{8-r_{2}}}(\tau)
$$

The $g$-action for another $E_{8}$-factor is defined in the same way with an integer $r_{3}$ $\left(0 \leq r_{3} \leq 8\right)$.

\subsection{Construction of heterotic T-folds}

Now, let us discuss how to construct the full building blocks characterized by the three integers $\left(r_{1}, r_{2}, r_{3}\right)$, which are modular covariant. In other words, we would like to construct the modular completions of (5.5) and (5.9). For this purpose we recall the modular covariant blocks (4.8), (4.10), (4.12), and consider their extensions to the $r$-dim. torus $T^{r}\left[X_{r}\right]$ composed of their products. For the 'odd sector' with $a \in 2 \mathbb{Z}+1$ or $b \in 2 \mathbb{Z}+1$, they are organized into the form,

$$
Z_{(a, b)}^{T^{r}\left[X_{r}\right]}(\tau):=\epsilon_{(a, b)}^{[r]} \overline{\left[\tilde{\chi}_{(a, b)}^{A_{1}}(\tau)\right]^{r}} \chi_{(a, b)}^{X_{r}}(\tau) .
$$

Here $\epsilon_{(a, b)}^{[r]}$ denotes the phase factor assuring the modular covariance, which can be directly read off from (4.8), (4.10), (4.12), and generally expressed as

$$
\epsilon_{(a, b)}^{[r]}:=e^{\frac{i \pi}{8} r(-1)^{a} a b}\left(\kappa_{(a, b)}\right)^{r}, \quad(a \in 2 \mathbb{Z}+1 \text { or } b \in 2 \mathbb{Z}+1)
$$

with

$$
\kappa_{(a, b)}:= \begin{cases}-1 & a \equiv 3,5(\bmod 8), b \in 2 \mathbb{Z}+1, \\ 1 & \text { otherwise }\end{cases}
$$

Note that the peculiar factor $\kappa_{(a, b)}$ affects only for odd $r$.

\footnotetext{
${ }^{6}$ The uniqueness of the decomposition (5.8) would be slightly non-trivial, even though it is almost trivial for the $D_{4}$-case (5.3). For instance, in the case of $r_{2}=1$, one might think that another decomposition

$$
\Lambda_{E_{8}}=\Lambda_{(0)}^{A_{1}} \oplus\left(\Lambda_{(0)}^{A_{1}} \oplus \Lambda_{(0)}^{D_{6}}\right)+\Lambda_{\mathrm{rem}},
$$

would be allowed. However, $\Lambda_{\text {rem }}$ here includes the conjugacy class such as $\Lambda_{(0)}^{A_{1}} \oplus\left(\Lambda_{(1)}^{A_{1}} \oplus \Lambda_{(s)}^{D_{6}}\right)$. Thus, this possibility is excluded by the condition (ii), and we obtain the unique decomposition with $X_{7}=E_{7}$.
} 
Furthermore, it is useful to note the following observations:

- The function $\left|\tilde{\chi}_{(a, b)}^{A_{1}}(\tau)\right|^{2}$ satisfies the modular covariance of the form (3.6).

- The function $(-1)^{a b}\left[\tilde{\chi}_{(a, b)}^{A_{1}}(\tau)\right]^{8}$ is similarly modular covariant up to the phases arising from the $T$-transformation acting on the factor $\eta(\tau)^{-8}$.

Based on these facts, one can construct the building blocks with the expected modular properties as follows:

(1) $\boldsymbol{T}^{\mathbf{4}}\left[\boldsymbol{D}_{4}\right]$-sector. Fix an integer $r(0 \leq r \leq 4)$, and set

$$
\begin{aligned}
F_{(a, b)}^{[r]}(\tau) & :=Z_{(a, b)}^{T^{r}\left[X_{r}\right]}(\tau) \cdot\left|\tilde{\chi}_{(a, b)}^{A_{1}}(\tau)\right|^{2(4-r)} \\
& \equiv \epsilon_{(a, b)}^{[r]} \overline{\left[\tilde{\chi}_{(a, b)}^{A_{1}}(\tau)\right]^{4}}\left[\tilde{\chi}_{(a, b)}^{A_{1}}(\tau)\right]^{4-r} \chi_{(a, b)}^{X_{r}}(\tau) .
\end{aligned}
$$

By construction, $F_{(a, b)}^{[r]}(\tau)$ is obviously modular covariant as

$$
\left.F_{(a, b)}^{[r]}(\tau)\right|_{T}=F_{(a, a+b)}^{[r]}(\tau),\left.\quad F_{(a, b)}^{[r]}(\tau)\right|_{S}=F_{(b,-a)}^{[r]}(\tau) .
$$

(2) $\boldsymbol{E}_{\mathbf{8}} \times \boldsymbol{E}_{\mathbf{8}}$-sector. For a single $E_{8}$-factor, fix an integer $s(0 \leq s \leq 8)$, and define a chiral building block as

$$
\begin{aligned}
G_{(a, b)}^{[s]}(\tau) & :=Z_{(a, b)}^{T^{r}\left[X_{s}\right]}(\tau) \cdot\left|\tilde{\chi}_{(a, b)}^{A_{1}}(\tau)\right|^{-2 s} \cdot(-1)^{a b}\left[\tilde{\chi}_{(a, b)}^{A_{1}}(\tau)\right]^{8} \\
& \equiv \epsilon_{(a, b)}^{[s]}(-1)^{a b}\left[\tilde{\chi}_{(a, b)}^{A_{1}}(\tau)\right]^{8-s} \chi_{(a, b)}^{X_{s}}(\tau) .
\end{aligned}
$$

Then, $G_{(a, b)}^{[s]}(\tau)$ is 'almost' modular covariant, which precisely means the following modular properties,

$$
\left.G_{(a, b)}^{[s]}(\tau)\right|_{T}=e^{-2 \pi i \frac{1}{3}} G_{(a, a+b)}^{[s]}(\tau),\left.\quad G_{(a, b)}^{[s]}(\tau)\right|_{S}=G_{(b,-a)}^{[s]}(\tau) .
$$

Chiral blocks for another $E_{8}$-factor are identical.

To describe the total modular invariant, we still need to describe the free fermion chiral block in the right-mover, which is twisted by $\left(-\mathbf{1}_{R}\right)^{\otimes 4}$. This has been presented e.g. in $[16,17]$, and can be concisely expressed as

$$
\overline{f_{(a, b)}(\tau)}=(-1)^{a b} \epsilon_{(a, b)}^{[4]} \overline{\left[\left(\tilde{\chi}_{(a, b)}^{A_{1}}(\tau)\right)^{4}-\left(\tilde{\chi}_{(a, b)}^{A_{1}}(\tau)\right)^{4}\right]} \quad(a \in 2 \mathbb{Z}+1 \text { or } b \in 2 \mathbb{Z}+1),
$$

in terms of the notation adopted here. Here the trivial cancellation appearing in the bracket $[\cdots]$ just means the existence of supersymmetry. A more explicit form of $f_{(a, b)}(\tau)$ is given in appendix A.2. The modularity of $\overline{f_{(a, b)}(\tau)}$ is expressed as

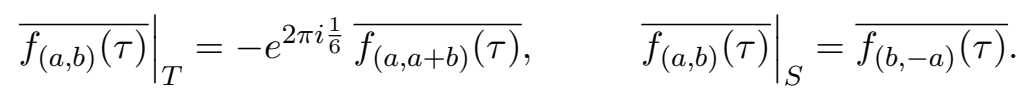


Combining all the sectors, we can write down the total partition function characterized by three integers $\left(r_{1}, r_{2}, r_{3}\right)\left(0 \leq r_{1} \leq 4,0 \leq r_{2}, r_{3} \leq 8\right)$ as follows:

$$
Z^{\left[r_{1}, r_{2}, r_{3}\right]}(\tau)=\frac{1}{2} Z^{5 d}(\tau) \sum_{w, m \in \mathbb{Z}} Z_{R,(w, m)}(\tau) Z_{(w, m)}^{\left[r_{1}, r_{2}, r_{3}\right]}(\tau)
$$

where

$$
Z_{(w, m)}^{\left[r_{1}, r_{2}, r_{3}\right]}(\tau):=\overline{\left[\left(\frac{\theta_{3}}{\eta}\right)^{4}-\left(\frac{\theta_{4}}{\eta}\right)^{4}-\left(\frac{\theta_{2}}{\eta}\right)^{4}\right]} \sum_{j}\left|\chi_{j}^{D_{4}}(\tau)\right|^{2}\left[\chi_{0}^{E_{8}}(\tau)\right]^{2},
$$

for the 'even sector' $(w, m \in 2 \mathbb{Z})$, which is actually independent of $\left(r_{1}, r_{2}, r_{3}\right)$, and

$$
\begin{aligned}
& Z_{(w, m)}^{\left[r_{1}, r_{2}, r_{3}\right]}(\tau):=\overline{f_{(w, m)}(\tau)} F_{(w, m)}^{\left[4-r_{1}\right]}(\tau) G_{(w, m)}^{\left[8-r_{2}\right]}(\tau) G_{(w, m)}^{\left[8-r_{3}\right]}(\tau) \\
& \equiv \epsilon_{(w, m)}^{\left[-\sum_{i} r_{i}\right]} \overline{\left[\left(\tilde{\chi}_{(w, m)}^{A_{1}}(\tau)\right)^{4}-\left(\tilde{\chi}_{(w, m)}^{A_{1}}(\tau)\right)^{4}\right]} \overline{\left[\tilde{\chi}_{(w, m)}^{A_{1}}(\tau)\right]^{4}} \\
& \quad \times\left[\tilde{\chi}_{(w, m)}^{A_{1}}(\tau)\right]^{\sum_{i} r_{i}} \chi_{(w, m)}^{X_{4-r_{1}}}(\tau) \prod_{i=2,3} \chi_{(w, m)}^{X_{8-r_{i}}(\tau),}
\end{aligned}
$$

for the 'odd sector' ( $w \in 2 \mathbb{Z}+1$ or $m \in 2 \mathbb{Z}+1)$. In (5.19), we denote the (transverse part of) bosonic sector of the $X^{0,1, \ldots, 4}$-directions as $Z_{5 \mathrm{~d}}(\tau)$, which is assumed to be modular invariant and not important here. The modular invariance of the total partition function $Z^{\left[r_{1}, r_{2}, r_{3}\right]}(\tau)$ is now obvious due to the modular covariance of the building blocks $Z_{(w, m)}^{\left[r_{1}, r_{2}, r_{3}\right]}(\tau)$. Especially the covariance of the odd sector (5.21) is readily confirmed by the relations (5.14), (5.16) and (5.18).

We add a few comments:

- In the cases when all $r_{i}$ are even, only the $D_{2 r}$-lattices (or the $E_{8}$-lattice itself) come into the above construction. For these cases, our heterotic T-fold vacua can be reproduced by the free fermion construction. However, when at least one of $r_{i}$ is odd, our construction does not reduce to the free fermion construction.

- It is straightforward to apply the above construction to the case of $T^{4}\left[D_{2} \times D_{2}\right]$ $\left(\equiv T^{4}\left[\left(A_{1}\right)^{4}\right]\right)$. The lattice decomposition (5.3) should be replaced with

$$
\Lambda_{(\alpha)}^{D_{2} \times D_{2}}=\sum_{\left\{\alpha_{i}\right\}, \beta}\left[\Lambda_{\left(\alpha_{1}\right)}^{A_{1}} \oplus \cdots \oplus \Lambda_{\left(\alpha_{r_{1}}\right)}^{A_{1}}\right] \oplus \Lambda_{(\beta)}^{Y_{4-r_{1}}}
$$

and the root system $Y_{r}(0 \leq r \leq 4)$ is uniquely determined as for $T^{4}\left[D_{4}\right]$. The result is explicitly listed in table 2. Therefore, in order to construct the desired vacua, we only have to replace $\chi_{(w, m)}^{X_{4-r_{1}}}(\tau)$ with $\chi_{(w, m)}^{Y_{4-r_{1}}}(\tau)$ in $(5.21)$, and also $\chi_{j}^{D_{4}}(\tau)$ with $\left[\chi_{j}^{D_{2}}(\tau)\right]^{2}$ in (5.20). 


\begin{tabular}{|c|c|c|c|c|c|}
\hline$r$ & 0 & 1 & 2 & 3 & 4 \\
\hline$Y_{r}$ & trivial & $A_{1}$ & $D_{2}$ & $A_{1} D_{2}$ & $D_{2} D_{2}$ \\
\hline
\end{tabular}

Table 2. list of $Y_{r}$.

\subsection{Unitarity in each winding sector}

In the heterotic string vacua we constructed above, the action of the orbifold twist $\sigma=$ $\mathcal{T}_{2 \pi R} \otimes g$ is simple in the untwisted sector, namely, the unwound sector along $S_{R}^{1}$, because $g$ is involutive on the untwisted Hilbert space, $g^{2}=\mathbf{1}$. However, the situation gets much more complicated in the twisted sectors, especially in the winding sectors with odd winding $w \in 2 \mathbb{Z}+1$ due to the existence of the non-trivial phase factor $\epsilon_{(w, m)}^{[*]}$ given in (5.11). It is thus not so obvious whether or not the string spectrum is unitary in each winding sector, which is read off by the standard technique of the Poisson resummation with respect to the temporal winding $m$. Namely, after summing over $m$ and rewriting the total partition function in the form,

$$
Z^{\left[r_{1}, r_{2}, r_{3}\right]}(\tau)=Z^{5 d}(\tau) \sum_{w \in \mathbb{Z}}\left[Z_{w}^{(\mathrm{NS})}(\tau)+Z_{w}^{(\mathrm{R})}(\tau)\right](\equiv 0)
$$

each of $Z_{w}^{(\mathrm{NS})}(\tau)$ should be $q^{-}$(and $\bar{q}_{-}$) expanded with coefficients belonging to $\mathbb{Z}_{\geq 0}$.

Although it would look more cumbersome because of the complexity of the phase factor $\epsilon_{(w, m)}^{[*]}$, we can perform the Poisson resummation analysis in a manner following $[14,16,17]$. After that, we can confirm that the above heterotic vacua are indeed unitary for an arbitrary choice of $\left(r_{1}, r_{2}, r_{3}\right)$. We here briefly sketch how it works as follows:

- For the sectors with $w \in 2 \mathbb{Z}$, it is easy to see the spectrum is unitary. Indeed, since the fermion chiral block $\overline{f_{(a, b)}(\tau)}$ given in (5.17) (or (A.19) for a more explicit form) with $a \in 2 \mathbb{Z}, b \in 2 \mathbb{Z}+1$ vanishes because of the cancellation only within the NS-sector, we find

$$
Z_{w}^{(\mathrm{NS})}(\tau)=\left.Z_{w}^{(\mathrm{NS})}(\tau)\right|_{\text {even } m},\left.\quad Z_{w}^{(\mathrm{NS})}(\tau)\right|_{\text {odd } m} \equiv 0, \quad\left({ }^{\forall} w \in 2 \mathbb{Z}\right),
$$

where $\left.Z_{w}^{(\mathrm{NS})}(\tau)\right|_{\text {even } m}\left(\left.Z_{w}^{(\mathrm{NS})}(\tau)\right|_{\text {odd } m}\right)$ denotes the summation over the even (odd) temporal winding $m$. The remaining $\left.Z_{w}^{(\mathrm{NS})}(\tau)\right|_{\text {even } m}$ is then $q$-expanded in the desired form thanks to the absence of the phase factor $\epsilon_{(w, m)}^{[*]}$ in (5.20).

- For the sectors with $w \in 2 \mathbb{Z}+1$, we have

$$
\begin{aligned}
Z_{w}^{(\mathrm{NS})}(\tau) & =\left.Z_{w}^{(\mathrm{NS})}(\tau)\right|_{\text {even } m}+\left.Z_{w}^{(\mathrm{NS})}(\tau)\right|_{\text {odd } m} \\
& =\left.Z_{w}^{(\mathrm{NS})}(\tau)\right|_{\text {even } m}+\left.Z_{w}^{(\mathrm{NS})}(\tau+1)\right|_{\text {even } m}
\end{aligned}
$$

The equality in the second line follows just because of the covariance of total building blocks under the modular $T$-transformation. 
- Nextly, we evaluate $\left.Z_{w}^{(\mathrm{NS})}(\tau)\right|_{\text {even } m},(w \in 2 \mathbb{Z}+1)$ by using the Poisson resummation. The relevant computation is now straightforward, since the phase factor $\epsilon_{(w, m)}^{[*]}$ is relatively simple, $\epsilon_{\left(w, 2 m^{\prime}\right)}^{[-\bar{r}]}=e^{i \pi \frac{\bar{r}}{4} w m^{\prime}}$, where we set $\bar{r} \equiv \sum_{i} r_{i}$. Other types of phase factors may come from $\chi_{\left(w, 2 m^{\prime}\right)}^{X_{r}}(\tau)$ as in (A.13), (A.14) and (A.15). In any case, however, the relevant phase factors always have the form such as $e^{2 \pi i \alpha m^{\prime}}$ with some rational number $\alpha$. This yields the shift of the KK momentum, $\frac{1}{2 R} n \rightarrow \frac{1}{2 R}(n+\alpha)$, and no extra phases are left. We thus obtain the $q$-expansion with positive coefficients belonging to $\frac{1}{2} \mathbb{Z} .^{7}$

- Finally, we pick up the remaining sector, $\left.\left.Z_{w}^{(\mathrm{NS})}(\tau)\right|_{\text {odd } m} \equiv\left[\left.Z_{w}^{(\mathrm{NS})}(\tau)\right|_{\operatorname{even} m}\right]\right|_{T}$. As pointed out in [14], this is Poisson resummed into almost the same form as $\left.Z_{w}^{(\mathrm{NS})}(\tau)\right|_{\text {even } m}$, but with an extra minus sign in each term with the level mismatch $h-\tilde{h} \in \frac{1}{2}+\mathbb{Z}$. In the end, we conclude that the total partition sum for the odd winding sector (5.25) is indeed $q$-expanded only with the coefficients belonging to $\mathbb{Z}_{\geq 0}$.

\section{Conclusions}

We demonstrated that one can systematically construct the modular invariant partition functions for the T-fold CFTs by using the Lie algebra lattices. We first discussed the case of bosonic strings. By the condition that the background moduli is at a fixed point for a simple T-duality transformation realized as a chiral reflection, the possible Lie algebras for the Englert-Neveu lattices are restricted to the four cases listed in (4.6) among simple simply-laced ones. Based on the fact that the characters of the level-one affine Lie algebras form a finite dimensional representation of the modular group, the partition functions for the fiber torus part are found to satisfy the modular covariance of the form (3.7). The results are listed in (4.8), (4.10), (4.12) and (4.14). Together with the base part, summing up these gives the desired modular invariants for T-folds. Similar constructions are possible also by using the Euclidean even self-dual lattices.

We then applied the above construction to the T-folds in the $E_{8} \times E_{8}$ heterotic string theory. As an example, we took a fiber torus representing the $D_{4}$ Englert-Neveu lattice. Incorporating the non-trivial twists/phases in the left-moving sector, we obtained a class of modular invariant partition functions of the T-fold CFTs which are labeled by three integers. In the twisted sectors, the partition functions in the left-mover are given by the building blocks obtained in the bosonic-string case, which are composed of the characters of the affine Lie algebras at level one. After the Poisson resummation, one can also check the unitarity of the spectrum. The case of the $D_{2} \times D_{2}$ torus was briefly discussed.

Our construction in the bosonic-string case formally resembles the truncation of the bosonic-string spectrum to the heterotic-string spectrum, which is used to study the Tduality of the latter $[26,32]$. Indeed, one can start with a $\left(d+d^{\prime}\right)$-dimensional torus whose

\footnotetext{
${ }^{7}$ The potential factor $1 / 2$ in the coefficients of $q$-expansion comes from the fact that the Poisson resummation is now made over $m \in 2 \mathbb{Z}$ rather than $m \in \mathbb{Z}$.
} 
background moduli takes the same form as in the truncation,

$$
E_{I J}=\left(\begin{array}{lc}
E_{i j} & 0 \\
A_{\mu j} & E_{\mu \nu}+\frac{1}{4} A_{\mu i} A_{\nu j} G^{i j}
\end{array}\right) \quad\left(i, j=1, \ldots, d, \quad \mu, \nu=1, \ldots, d^{\prime}\right),
$$

and proceed as in section 3 and 4. An interesting possibility in this case is that the additional moduli $A_{\mu k}$ may be incorporated in the T-fold CFTs. For this to be the case, one needs to check the fixed-point condition of the T-duality and also to confirm that the twisted partition functions with non-trivial $A_{\mu i}$ indeed satisfy the modular covariance of the form (3.7). We leave these as future problems.

It is worthwhile to remark that the heterotic T-folds we constructed include novel cases which contain rather non-trivial phase factors and are not reduced to the free fermion construction. It would thus be interesting to apply our construction to building the 'realistic' heterotic vacua of asymmetric orbifolds, since recent attempts so far are mainly based on the free fermion construction e.g. as in [11] for the SUSY vacua and in [36-42] for the SUSY-breaking ones. Especially, it is indeed possible to extend the present construction to a variety of the non-SUSY heterotic T-folds by following $[16,17]$. It would also be interesting to figure out the moduli space of such a class of vacua. We would like to return to these issues in a future work.

\section{Acknowledgments}

We would like to thank C. Ahn and Z. Bajnok for useful comments. This work is supported in part by JSPS Grant-in-Aid for Scientific Research 24540248 and Japan-Hungary Research Cooperative Program from Japan Society for the Promotion of Science (JSPS).

\section{A Summary of building blocks}

In this appendix we summarize the definitions of the building blocks that are repeatedly used in the main text.

\section{A.1 Building blocks associated with Lie algebra lattices}

As preparation, we summarize our conventions of theta functions and the character formulas of affine Lie algebras relevant to our analysis.

Theta functions. Our convention of theta functions are

$$
\begin{aligned}
& \theta_{1}(\tau, z):=i \sum_{n=-\infty}^{\infty}(-1)^{n} q^{(n-1 / 2)^{2} / 2} y^{n-1 / 2} \equiv 2 \sin (\pi z) q^{1 / 8} \prod_{m=1}^{\infty}\left(1-q^{m}\right)\left(1-y q^{m}\right)\left(1-y^{-1} q^{m}\right), \\
& \theta_{2}(\tau, z):=\sum_{n=-\infty}^{\infty} q^{(n-1 / 2)^{2} / 2} y^{n-1 / 2} \equiv 2 \cos (\pi z) q^{1 / 8} \prod_{m=1}^{\infty}\left(1-q^{m}\right)\left(1+y q^{m}\right)\left(1+y^{-1} q^{m}\right),
\end{aligned}
$$




$$
\begin{aligned}
& \theta_{3}(\tau, z):=\sum_{n=-\infty}^{\infty} q^{n^{2} / 2} y^{n} \equiv \prod_{m=1}^{\infty}\left(1-q^{m}\right)\left(1+y q^{m-1 / 2}\right)\left(1+y^{-1} q^{m-1 / 2}\right) \\
& \theta_{4}(\tau, z):=\sum_{n=-\infty}^{\infty}(-1)^{n} q^{n^{2} / 2} y^{n} \equiv \prod_{m=1}^{\infty}\left(1-q^{m}\right)\left(1-y q^{m-1 / 2}\right)\left(1-y^{-1} q^{m-1 / 2}\right) \\
& \eta(\tau):=q^{1 / 24} \prod_{n=1}^{\infty}\left(1-q^{n}\right)
\end{aligned}
$$

where $q:=e^{2 \pi i \tau}, y:=e^{2 \pi i z}$. We use abbreviations, $\theta_{i}(\tau) \equiv \theta_{i}(\tau, 0)$ with $\theta_{1}(\tau) \equiv 0$.

Characters of $\left(\widehat{A}_{\mathbf{1}}\right)_{\mathbf{1}}$. The character of affine $A_{1}$ of level one $\left(\left(\widehat{A}_{1}\right)_{1}\right)$ is written as

$$
\left.\chi_{0}^{A_{1}}(\tau):=\frac{\theta_{3}(2 \tau)}{\eta(\tau)} \quad \text { (basic rep. }\right), \quad \chi_{1}^{A_{1}}(\tau):=\frac{\theta_{2}(2 \tau)}{\eta(\tau)} \quad(\text { spin } 1 / 2 \text { rep. }) .
$$

We also define

$$
\chi_{ \pm}^{A_{1}}(\tau):=\chi_{0}^{A_{1}}(\tau) \pm \chi_{1}^{A_{1}}(\tau)
$$

Characters of $\left(\widehat{D}_{\boldsymbol{r}}\right)_{1} \quad\left(\boldsymbol{r} \in \mathbf{2} \mathbb{Z}_{>\mathbf{0}}\right)$. The characters of $\left(\widehat{D}_{r}\right)_{1}$ are given by

$$
\begin{array}{ll}
\chi_{0}^{D_{r}}(\tau):=\frac{1}{2 \eta(\tau)^{r}}\left[\theta_{3}(\tau)^{r}+\theta_{4}(\tau)^{r}\right] \quad \text { (basic rep.) }, \\
\chi_{v}^{D_{r}}(\tau):=\frac{1}{2 \eta(\tau)^{r}}\left[\theta_{3}(\tau)^{r}-\theta_{4}(\tau)^{r}\right] \quad \text { (vector rep.) }, \\
\chi_{s}^{D_{r}}(\tau) \equiv \chi_{c}^{D_{r}}(\tau):=\frac{\theta_{2}(\tau)^{r}}{2 \eta(\tau)^{r}} \quad \text { (spinor and cospinor rep.). }
\end{array}
$$

Characters $\left(\widehat{\boldsymbol{E}}_{\mathbf{7}}\right)_{\mathbf{1}}$. The character of $\left(\widehat{E}_{7}\right)_{1}$ is given by

$$
\chi_{0}^{E_{7}}(\tau):=\frac{1}{2 \eta(\tau)^{7}}\left[\theta_{3}(2 \tau)\left(\theta_{3}(\tau)^{6}+\theta_{4}(\tau)^{6}\right)+\theta_{2}(2 \tau) \theta_{2}(\tau)^{6}\right]
$$

for the basic representation, and by

$$
\chi_{1}^{E_{7}}(\tau):=\frac{1}{2 \eta(\tau)^{7}}\left[\theta_{2}(2 \tau)\left(\theta_{3}(\tau)^{6}-\theta_{4}(\tau)^{6}\right)+\theta_{3}(2 \tau) \theta_{2}(\tau)^{6}\right]
$$

for the fundamental representation $\mathbf{5 6}$ with conformal weight $h=\frac{3}{4}$. We also define

$$
\chi_{ \pm}^{E_{7}}(\tau):=\chi_{0}^{E_{7}}(\tau) \pm \chi_{1}^{E_{7}}(\tau)
$$

Character of $\left(\widehat{\boldsymbol{E}}_{\mathbf{8}}\right)_{\mathbf{1}}$. The root lattice of $E_{8}$ is the simplest example of even self-dual lattices, and the corresponding chiral block for strings is the character of the basic representation of $\left(\widehat{E}_{8}\right)_{1}$,

$$
\chi_{0}^{E_{8}}(\tau):=\frac{1}{2 \eta(\tau)^{8}}\left[\theta_{3}(\tau)^{8}+\theta_{4}(\tau)^{8}+\theta_{2}(\tau)^{8}\right] .
$$


Now, we summarize the functions used in order to compose the modular covariant blocks in the main text, which are associated with the Lie algebra lattices for $A_{1}, D_{r}(r \in$ $\left.2 \mathbb{Z}_{>0}\right), E_{7}, E_{8}$ :

$$
\begin{aligned}
& \chi_{(a, b)}^{D_{r}}(\tau):= \begin{cases}\frac{1}{2 \eta(\tau)^{r}}\left\{\theta_{3}(\tau)^{r}+e^{\frac{i \pi r}{4} a} \theta_{4}(\tau)^{r}\right\} & (a \in 2 \mathbb{Z}, b \in 2 \mathbb{Z}+1), \\
\frac{1}{2 \eta(\tau)^{r}}\left\{\theta_{3}(\tau)^{r}+e^{\frac{i \pi r}{4} b} \theta_{2}(\tau)^{r}\right\} & (a \in 2 \mathbb{Z}+1, b \in 2 \mathbb{Z}), \\
\frac{1}{2 \eta(\tau)^{r}}\left\{\theta_{4}(\tau)^{r}+e^{\frac{i \pi r}{4}(a+b-1)} \theta_{2}(\tau)^{r}\right\} & (a \in 2 \mathbb{Z}+1, b \in 2 \mathbb{Z}+1),\end{cases} \\
& \chi_{(a, b)}^{A_{1}}(\tau):= \begin{cases}\frac{1}{2}\left\{\chi_{+}^{A_{1}}(\tau)+e^{\frac{i \pi}{2} a} \chi_{-}^{A_{1}}(\tau)\right\} & (a \in 2 \mathbb{Z}, b \in 2 \mathbb{Z}+1), \\
\frac{1}{\sqrt{2}}\left\{\chi_{0}^{A_{1}}(\tau)+e^{\frac{i \pi}{2} b} \chi_{1}^{A_{1}}(\tau)\right\} & (a \in 2 \mathbb{Z}+1, b \in 2 \mathbb{Z}), \\
\frac{1}{\sqrt{2}}\left\{\chi_{0}^{A_{1}}(\tau)+e^{\frac{i \pi}{2}(a+b-1)} \chi_{1}^{A_{1}}(\tau)\right\} & (a \in 2 \mathbb{Z}+1, b \in 2 \mathbb{Z}+1),\end{cases} \\
& \chi_{(a, b)}^{E_{7}}(\tau):= \begin{cases}\frac{1}{2}\left\{\chi_{+}^{E_{7}}(\tau)+e^{-\frac{i \pi}{2} a} \chi_{-}^{E_{7}}(\tau)\right\} & (a \in 2 \mathbb{Z}, b \in 2 \mathbb{Z}+1), \\
\frac{1}{\sqrt{2}}\left\{\chi_{0}^{E_{7}}(\tau)+e^{-\frac{i \pi}{2} b} \chi_{1}^{E_{7}}(\tau)\right\} & (a \in 2 \mathbb{Z}+1, b \in 2 \mathbb{Z}), \\
\frac{1}{\sqrt{2}}\left\{\chi_{0}^{E_{7}}(\tau)+e^{-\frac{i \pi}{2}(a+b-1)} \chi_{1}^{E_{7}}(\tau)\right\} & (a \in 2 \mathbb{Z}+1, b \in 2 \mathbb{Z}+1),\end{cases} \\
& \chi_{(a, b)}^{E_{8}}(\tau):=(-1)^{a b} \chi_{0}^{E_{8}}(\tau), \quad(a \in 2 \mathbb{Z}+1 \text { or } b \in 2 \mathbb{Z}+1) \text {. }
\end{aligned}
$$

In each case, the integer labels $a, b$ characterize the spatial and temporal boundary conditions. We also denote

$$
\tilde{\chi}_{(a, b)}^{A_{1}}(\tau):= \begin{cases}\frac{1}{\eta(\tau)} \sum_{n \in \mathbb{Z}}(-1)^{n} q^{n^{2}} \equiv \sqrt{\frac{\theta_{3}(\tau) \theta_{4}(\tau)}{\eta(\tau)^{2}}} & (a \in 2 \mathbb{Z}, b \in 2 \mathbb{Z}+1), \\ \frac{\sqrt{2}}{\eta(\tau)} \sum_{n \in \mathbb{Z}} q^{\left(n+\frac{1}{4}\right)^{2}} \equiv \sqrt{\frac{\theta_{3}(\tau) \theta_{2}(\tau)}{\eta(\tau)^{2}}} & (a \in 2 \mathbb{Z}+1, b \in 2 \mathbb{Z}), \\ \frac{\sqrt{2}}{\eta(\tau)} \sum_{n \in \mathbb{Z}}(-1)^{n} q^{\left(n+\frac{1}{4}\right)^{2}} \equiv \sqrt{\frac{\theta_{4}(\tau) \theta_{2}(\tau)}{\eta(\tau)^{2}}} & (a \in 2 \mathbb{Z}+1, b \in 2 \mathbb{Z}+1),\end{cases}
$$

for the $\left(\widehat{A}_{1}\right)_{1}$-characters twisted by the involution $\rho_{A_{1}} \equiv e^{-\frac{i \pi}{2} \ell} e^{i \pi J_{0}^{3}}$ in (5.4). See e.g. appendix $\mathrm{C}$ of [8] for more detail. These coincide with $\vartheta_{p q}^{1 / 2}$ defined in (3.18). It is easy to confirm the equality

$$
\chi_{(a, b)}^{D_{2}}(\tau)=\left[\chi_{(a, b)}^{A_{1}}(\tau)\right]^{2},
$$

which is consistent with the isomorphism of the root lattices $\Lambda_{D_{2}} \cong \Lambda_{A_{1}} \oplus \Lambda_{A_{1}}$. We also use the notation such as $\chi_{(a, b)}^{X_{3}}(\tau) \equiv \chi_{(a, b)}^{A_{1}}(\tau) \chi_{(a, b)}^{D_{2}}(\tau)$ for $X_{3}=A_{1} D_{2}$ (see table 1).

\section{A.2 Free fermion chiral blocks}

We next describe the chiral blocks for the 8 world-sheet fermions $\psi_{L}^{i}$ twisted by

$$
\left(-\mathbf{1}_{L}\right)^{\otimes 4}: \psi_{L}^{i} \longmapsto-\psi_{L}^{i}, \quad(i=6, \ldots, 9) .
$$


The relevant blocks are explicitly written as

$$
\begin{aligned}
f_{(a, b)}(\tau) & :=2 q^{\frac{1}{4} a^{2}} e^{\frac{i \pi}{2} a b}\left(\frac{\theta_{1}\left(\tau, \frac{a \tau+b}{2}\right)}{\eta(\tau)}\right)^{2}\left(\frac{\theta_{1}(\tau, 0)}{\eta(\tau)}\right)^{2} \\
& \equiv(-1)^{a b} e^{\frac{i \pi}{2} a b}\left[\left(\tilde{\chi}_{(a, b)}^{A_{1}}(\tau)\right)^{4}-\left(\tilde{\chi}_{(a, b)}^{A_{1}}(\tau)\right)^{4}\right] \\
& \equiv \begin{cases}e^{\frac{i \pi}{2} a b}\left\{\left(\frac{\theta_{3}}{\eta}\right)^{2}\left(\frac{\theta_{4}}{\eta}\right)^{2}-\left(\frac{\theta_{4}}{\eta}\right)^{2}\left(\frac{\theta_{3}}{\eta}\right)^{2}+0\right\} & (a \in 2 \mathbb{Z}, b \in 2 \mathbb{Z}+1), \\
e^{\frac{i \pi}{2} a b}\left\{\left(\frac{\theta_{3}}{\eta}\right)^{2}\left(\frac{\theta_{2}}{\eta}\right)^{2}+0-\left(\frac{\theta_{2}}{\eta}\right)^{2}\left(\frac{\theta_{3}}{\eta}\right)^{2}\right\} & (a \in 2 \mathbb{Z}+1, b \in 2 \mathbb{Z}), \\
-e^{\frac{i \pi}{2} a b}\left\{0+\left(\frac{\theta_{2}}{\eta}\right)^{2}\left(\frac{\theta_{4}}{\eta}\right)^{2}-\left(\frac{\theta_{4}}{\eta}\right)^{2}\left(\frac{\theta_{2}}{\eta}\right)^{2}\right\} & (a \in 2 \mathbb{Z}+1, b \in 2 \mathbb{Z}+1) .\end{cases}
\end{aligned}
$$

In the last line, each term corresponds to the NS, $\widetilde{N S}, \mathrm{R}$ sectors with keeping this order. These trivially vanish, as is consistent with the space-time SUSY. They satisfy the modular covariance of the form,

$$
\begin{aligned}
& \left.f_{(a, b)}(\tau)\right|_{S} \equiv f_{(a, b)}\left(-\frac{1}{\tau}\right)=f_{(b,-a)}(\tau), \\
& \left.f_{(a, b)}(\tau)\right|_{T} \equiv f_{(a, b)}(\tau+1)=-e^{-2 \pi i \frac{1}{6}} f_{(a, a+b)}(\tau) .
\end{aligned}
$$

Therefore, setting

$$
\mathcal{J}(\tau):=\left(\frac{\theta_{3}}{\eta}\right)^{4}-\left(\frac{\theta_{4}}{\eta}\right)^{4}-\left(\frac{\theta_{2}}{\eta}\right)^{4}
$$

we find

$$
\begin{aligned}
& {\left.\left[\overline{\mathcal{J}(\tau)} f_{(a, b)}(\tau)\right]\right|_{S} \equiv \overline{\mathcal{J}\left(-\frac{1}{\tau}\right)} f_{(a, b)}\left(-\frac{1}{\tau}\right)=\overline{\mathcal{J}(\tau)} f_{(b,-a)}(\tau),} \\
& {\left.\left[\overline{\mathcal{J}(\tau)} f_{(a, b)}(\tau)\right]\right|_{T} \equiv \overline{\mathcal{J}(\tau+1)} f_{(a, b)}(\tau+1)=\overline{\mathcal{J}(\tau)} f_{(a, a+b)}(\tau) .}
\end{aligned}
$$

Since (A.19) vanishes, the relations (A.20) may appear to be ambiguous. See, however, [16] for more precise arguments.

Open Access. This article is distributed under the terms of the Creative Commons Attribution License (CC-BY 4.0), which permits any use, distribution and reproduction in any medium, provided the original author(s) and source are credited.

\section{References}

[1] A. Dabholkar and C. Hull, Duality twists, orbifolds and fluxes, JHEP 09 (2003) 054 [hep-th/0210209] [INSPIRE].

[2] S. Hellerman, J. McGreevy and B. Williams, Geometric constructions of nongeometric string theories, JHEP 01 (2004) 024 [hep-th/0208174] [INSPIRE].

[3] A. Flournoy, B. Wecht and B. Williams, Constructing nongeometric vacua in string theory, Nucl. Phys. B 706 (2005) 127 [hep-th/0404217] [INSPIRE]. 
[4] C.M. Hull, A Geometry for non-geometric string backgrounds, JHEP 10 (2005) 065 [hep-th/0406102] [INSPIRE].

[5] C. Hull and B. Zwiebach, Double Field Theory, JHEP 09 (2009) 099 [arXiv: 0904.4664] [INSPIRE].

[6] A. Flournoy and B. Williams, Nongeometry, duality twists and the worldsheet, JHEP 01 (2006) 166 [hep-th/0511126] [INSPIRE].

[7] S. Hellerman and J. Walcher, Worldsheet CFTs for Flat Monodrofolds, hep-th/0604191 [INSPIRE].

[8] S. Kawai and Y. Sugawara, D-branes in T-fold conformal field theory, JHEP 02 (2008) 027 [arXiv:0709.0257] [INSPIRE].

[9] S. Kawai and Y. Sugawara, Mirrorfolds with K3 fibrations, JHEP 02 (2008) 065 [arXiv:0711.1045] [INSPIRE].

[10] P. Anastasopoulos, M. Bianchi, J.F. Morales and G. Pradisi, (Unoriented) T-folds with few T's, JHEP 06 (2009) 032 [arXiv:0901.0113] [INSPIRE].

[11] M. Bianchi, G. Pradisi, C. Timirgaziu and L. Tripodi, Heterotic T-folds with a small number of neutral moduli, JHEP 10 (2012) 089 [arXiv:1207.2665] [INSPIRE].

[12] C. Condeescu, I. Florakis and D. Lüst, Asymmetric Orbifolds, Non-Geometric Fluxes and Non-Commutativity in Closed String Theory, JHEP 04 (2012) 121 [arXiv:1202.6366] [INSPIRE].

[13] C. Condeescu, I. Florakis, C. Kounnas and D. Lüst, Gauged supergravities and non-geometric Q/R-fluxes from asymmetric orbifold CFT's, JHEP 10 (2013) 057 [arXiv:1307.0999] [INSPIRE].

[14] Y. Satoh and Y. Sugawara, Non-geometric Backgrounds Based on Topological Interfaces, JHEP 07 (2015) 022 [arXiv: 1502.05776] [INSPIRE].

[15] H.S. Tan, T-duality Twists and Asymmetric Orbifolds, JHEP 11 (2015) 141 [arXiv: 1508.04807] [INSPIRE].

[16] Y. Satoh, Y. Sugawara and T. Wada, Non-supersymmetric Asymmetric Orbifolds with Vanishing Cosmological Constant, JHEP 02 (2016) 184 [arXiv: 1512.05155] [INSPIRE].

[17] Y. Sugawara and T. Wada, More on Non-supersymmetric Asymmetric Orbifolds with Vanishing Cosmological Constant, JHEP 08 (2016) 028 [arXiv: 1605.07021] [INSPIRE].

[18] E. Wong and I. Affleck, Tunneling in quantum wires: A Boundary conformal field theory approach, Nucl. Phys. B 417 (1994) 403 [INSPIRE].

[19] V.B. Petkova and J.B. Zuber, Generalized twisted partition functions, Phys. Lett. B 504 (2001) 157 [hep-th/0011021] [INSPIRE].

[20] C. Bachas, J. de Boer, R. Dijkgraaf and H. Ooguri, Permeable conformal walls and holography, JHEP 06 (2002) 027 [hep-th/0111210] [INSPIRE].

[21] C. Bachas and I. Brunner, Fusion of conformal interfaces, JHEP 02 (2008) 085 [arXiv:0712.0076] [INSPIRE].

[22] Y. Satoh, On supersymmetric interfaces for string theory, JHEP 03 (2012) 072 [arXiv: 1112.5935] [INSPIRE]. 
[23] C. Bachas, I. Brunner and D. Roggenkamp, A worldsheet extension of $O(d, d: Z)$, JHEP 10 (2012) 039 [arXiv: 1205.4647] [InSPIRE].

[24] S. Elitzur, B. Karni, E. Rabinovici and G. Sarkissian, Defects, Super-Poincaré line bundle and Fermionic T-duality, JHEP 04 (2013) 088 [arXiv: 1301.6639] [INSPIRE].

[25] A. Dabholkar and C. Hull, Generalised T-duality and non-geometric backgrounds, JHEP 05 (2006) 009 [hep-th/0512005] [INSPIRE].

[26] A. Giveon, M. Porrati and E. Rabinovici, Target space duality in string theory, Phys. Rept. 244 (1994) 77 [hep-th/9401139] [INSPIRE].

[27] T. Kugo and B. Zwiebach, Target space duality as a symmetry of string field theory, Prog. Theor. Phys. 87 (1992) 801 [hep-th/9201040] [INSPIRE].

[28] J. Erler, Asymmetric orbifolds and higher level models, Nucl. Phys. B 475 (1996) 597 [hep-th/9602032] [INSPIRE].

[29] F. Englert and A. Neveu, Nonabelian Compactification of the Interacting Bosonic String, Phys. Lett. B 163 (1985) 349 [INSPIRE].

[30] W. Lerche, A.N. Schellekens and N.P. Warner, Lattices and Strings, Phys. Rept. 177 (1989) 1 [INSPIRE].

[31] S. Elitzur, E. Gross, E. Rabinovici and N. Seiberg, Aspects of Bosonization in String Theory, Nucl. Phys. B 283 (1987) 413 [inSPIRE].

[32] A. Giveon, E. Rabinovici and G. Veneziano, Duality in String Background Space, Nucl. Phys. B 322 (1989) 167 [INSPIRE].

[33] T. Eguchi and Y. Sugawara, Non-holomorphic Modular Forms and $\mathrm{SL}(2, \mathbb{R}) / U(1)$ Superconformal Field Theory, JHEP 03 (2011) 107 [arXiv: 1012.5721] [INSPIRE].

[34] K. Aoki, E. D'Hoker and D.H. Phong, On the construction of asymmetric orbifold models, Nucl. Phys. B 695 (2004) 132 [hep-th/0402134] [INSPIRE].

[35] O. King, A mass formula for unimodular lattices with no roots, Math. Comput. 72 (2003) 839, arXiv:math/0012231.

[36] M. Blaszczyk, S. Groot Nibbelink, O. Loukas and S. Ramos-Sanchez, Non-supersymmetric heterotic model building, JHEP 10 (2014) 119 [arXiv:1407.6362] [INSPIRE].

[37] C. Angelantonj, I. Florakis and M. Tsulaia, Universality of Gauge Thresholds in Non-Supersymmetric Heterotic Vacua, Phys. Lett. B 736 (2014) 365 [arXiv:1407.8023] [INSPIRE].

[38] C. Angelantonj, I. Florakis and M. Tsulaia, Generalised universality of gauge thresholds in heterotic vacua with and without supersymmetry, Nucl. Phys. B 900 (2015) 170 [arXiv: 1509.00027] [INSPIRE].

[39] A.E. Faraggi, C. Kounnas and H. Partouche, Large volume SUSY breaking with a solution to the decompactification problem, Nucl. Phys. B 899 (2015) 328 [arXiv:1410.6147] [INSPIRE].

[40] S. Abel, K.R. Dienes and E. Mavroudi, Towards a nonsupersymmetric string phenomenology, Phys. Rev. D 91 (2015) 126014 [arXiv:1502.03087] [INSPIRE].

[41] C. Kounnas and H. Partouche, Stringy $N=1$ super no-scale models, PoS (PLANCK 2015) 070 [arXiv: 1511.02709] [INSPIRE].

[42] C. Kounnas and H. Partouche, Super no-scale models in string theory, Nucl. Phys. B 913 (2016) 593 [arXiv: 1607.01767] [INSPIRE]. 